\title{
Analysis of oxylipins in human plasma: Comparison of ultrahigh-performance liquid chromatography and ultrahigh-performance supercritical fluid chromatography coupled to mass spectrometry
}

\author{
Róbert Berkecz $^{\mathrm{a}, \mathrm{b}}$, Miroslav Lísa ${ }^{\mathrm{a}}$, Michal Holčapek ${ }^{\mathrm{a}, *}$ \\ a Department of Analytical Chemistry, Faculty of Chemical Technology, University of Pardubice, Studentská 573, 53210 Pardubice, Czech Republic \\ ${ }^{\mathrm{b}}$ Department of Medical Chemistry, Faculty of Medicine, University of Szeged, Dóm tér 8, H-6720 Szeged, Hungary
}

\section{A R T I C L E I N F O}

\section{Article history:}

Received 23 January 2017

Received in revised form 23 June 2017

Accepted 27 June 2017

Available online 29 June 2017

\section{Keywords:}

Oxylipin

Eicosanoid

Prostaglandin

Plasma

UHPSFC/MS

UHPLC/MS

SPE

ESI

\begin{abstract}
A B S T R A C T
The potential of ultrahigh-performance liquid chromatography-mass spectrometry (UHPLC/MS) and ultrahigh-performance supercritical fluid chromatography (UHPSFC) coupled to negative-ion electrospray ionization mass spectrometry (ESI-MS) for the analysis of 46 oxylipins and 2 fatty acid standards is compared in terms of their chromatographic resolution with the emphasis on distinguishing isobaric interferences and the method sensitivity. UHPLC provides the baseline separation of 24 isobaric oxylipins within $13 \mathrm{~min}$, while UHPSFC enables the separation of only 20 isobaric oxylipins within $8 \mathrm{~min}$. Moreover, the UHPLC/ESI-MS method provides an average improvement of sensitivity by 3.5 -fold. A similar trend is observed in the analysis of human plasma samples, but lower ion suppression effects caused by lysophospholipids (LPL) are observed in case of UHPSFC/ESI-MS due to better separation of LPL. Both methods are fully applicable for the analysis of oxylipins, but UHPLC/ESI-MS method is preferred due to better separation and higher sensitivity, which results in the identification of 31 oxylipins in human plasma based on available standards and additional tentative 20 identifications based on accurate $\mathrm{m} / \mathrm{z}$ values and the fragmentation behavior known from the literature.
\end{abstract}

(C) 2017 Elsevier B.V. All rights reserved.

\section{Introduction}

Oxylipins, including eicosanoids, docosanoids, and octadecanoids, are an important family of lipids, which are formed by the oxidation of polyunsaturated fatty acids (PUFA) [1]. They are derived mainly from arachidonic acid (AA), docosahexaenoic acid, eicosapentaenoic acid and dihomo- $\gamma$-linolenic acid. PUFAs are mostly generated from glycerophospholipids (PL) by the enzyme phospholipase A2, and then they are further metabolized to eicosanoids through enzymatic processes by free separate enzyme families, such as cyclooxygenase (COX), lipoxygenase (LOX), and cytochrome P450 (CYP). In addition, PUFA can be autooxidized forming bioactive lipids via nonenzymatic pathways as well $[1,2]$. In humans, eicosanoids have a wide range of physiological effects in inflammation, cardiovascular protection, blood clotting, and apoptosis. The important biological roles and excretion of these lipid mediators into body fluids, such as blood, urine, and cerebrospinal

\footnotetext{
* Corresponding author.

E-mail address: Michal.Holcapek@upce.cz (M. Holčapek).
}

fluid, make them potential biomarker targets [2-6]. However, the concentration of eicosanoids ( $\mathrm{pmol} / \mathrm{mL}$ range) in human plasma is much lower compared to other endogenous lipids ( $\mathrm{nmol} / \mathrm{mL}$ range), such as glycerolipids, PL, sphingolipids, ceramides, and sterols.Therefore their analysis requires more sensitive analytical methods [7].

In past decades, the analysis of oxylipins was mainly performed by immunoassay techniques, such as radioimmunoassays (RIA) and enzyme immunoassays (EIA). The main disadvantage of these methods is the limited applicability for plasma and tissue samples due to immunological cross-reactivity and decreased sensitivity due to protein - eicosanoid interactions [2]. Gas chromatography-mass spectrometry (GC/MS) provides higher sensitivity and better selectivity than RIA and EIA, but the essential derivatization step during the sample preparation is time consuming and laborious [8,9]. Nowadays, liquid chromatography-mass spectrometry (LC/MS) is the most common technique used to simultaneously analyze eicosanoids and other oxylipins, especially in targeted tandem mass spectrometry (LC/MS/MS) mode using triple quadrupole $(\mathrm{QqQ})$ instruments with the selected reaction monitoring (SRM) scanning mode [8], because it provides accu- 
rate quantitative information due to high sensitivity and selectivity without derivatization step and the opportunity of online sample extraction [9]. Mass spectrometry plays a major role in the identification and quantitation of eicosanoids. Negative-ion electrospray ionization (ESI) is the most sensitive ionization mode for eicosanoids due to the presence of carboxylic acid [10]. The comprehensive nontargeted analysis of eicosanoids using quadrupole time-of-flight (QTOF) mass spectrometer provides more qualitative information on analyzed samples, such as the identification of new compounds, information on matrix or other endogenous compounds, while SRM scans on QqQ are better for sensitive quantitation [8-10].

The LC separation is the most widespread technique for the analysis of wide range of oxylipins. Reversed-phase (RP) mode performed mainly on octadecylsilica (C18) or octylsilica (C8) column provides the highest selectivity for the resolution of isobaric oxylipins $[2,6,8,9,11,12,14-18]$. Oxylipins are determined in various body fluids, such as amniotic fluid, cerebrospinal fluid, urine, serum, and plasma [9,11-18]. LC/MS/MS method has been applied for the simultaneous determination of 32 oxylipins in human plasma samples in 29 min using C8 column and ammonium formate - acetonitrile gradient [12]. Vreeken and coworkers have detected 36 human plasma oxylipins in $26 \mathrm{~min}$ by UHPLC/MS/MS method using C18 column and aqueous acetic acid - 2-propanol - acetonitrile gradient [16]. Recently, UHPLC/MS/MS method has been used to identify and quantify 60 endogenous oxylipins in human plasma using C18 column in 5 min using aqueous acetic acid - acetonitrile - 2-propanol gradient [17]. Faccioli and coworkers have profiled 22 oxylipins in human plasma within 30 min with UHPLC/MS/MS method using C18 column and aqueous acetic acid - acetonitrile 2-propanol gradient [18].

Supercritical fluid chromatography (SFC) successfully combines advantages of gas and liquid chromatography, and especially the novel approach based on the use of sub- $2 \mu \mathrm{m}$ particle columns yields ultrafast and efficient separations. This technique is referred as UHPSFC by the analogy to UHPLC, and may be easily coupled to mass spectrometry as well (UHPSFC/MS) [19]. Applications for various compounds classes illustrate the power of this new approach, e.g., example for synthetic cannabinoids [20], pharmaceutical compounds [21], phospholipids, and sphingolipids [22]. However, UHPSFC/MS method for the analysis of oxylipins has not been reported so far.

The main goal of this study is a comparison of UHPLC/ESI-MS and UHPSFC/ESI-MS methods in terms of their suitability for the analysis of wide range of oxylipins in human plasma samples using the set of 46 standards typically occurring in biological systems. All parameters are carefully optimized to achieve the best selectivity and sensitivity of the final method, which is then applied for the analysis of endogenous oxylipins in the human plasma extract.

\section{Experimental}

\subsection{Chemicals and standards}

Methanol, acetonitrile, 2-propanol, ethanol (all LC/MS grade), chloroform stabilized by $0.5-1 \%$ ethanol (HPLC grade), ammonium formate, ammonium acetate, formic acid, and acetic acid were purchased from Sigma-Aldrich (St. Louis, MO, USA). Deionized water was prepared with a Milli-Q Reference Water Purification System (Molsheim, France). Carbon dioxide 4.5 grade (99.995\%) was purchased from Messer Group Gmbh (Bad Soden, Germany).

8R-hydroxy-4Z,6E,10Z-hexadecatrienoic acid (tetranor12-HETE), 12S-hydroxy-5Z,8E,10E-heptadecatrienoic acid (12-HHTrE), 13-oxo-9Z,11E-octadecadienoic acid (13OxoODE), 9-oxo-10E,12Z-octadecadienoic acid (9-OxoODE),
13S-hydroxy-9Z,11E,15Z-octadecatrienoic acid (13-HOTrE), $( \pm)$ 13-hydroxy-9Z,11E-octadecadienoic acid (13-HODE), ( \pm 9-hydroxy-10E,12Z-octadecadienoic acid (9-HODE), ( \pm 12,13-dihydroxy-9Z-octadecenoic acid (12,13-DiHOME), $( \pm) 15$-hydroxy-5Z,8Z,11Z,13E,17Z-eicosapentaenoic acid (15HEPE), ( \pm )5-hydroxy-6E,8Z,11Z,14Z,17Z-eicosapentaenoic acid (5-HEPE), $( \pm) 11,(12)$-epoxy-5Z,8Z,14Z-eicosatrienoic acid (11,12EET), $( \pm) 5,6$-epoxy-8Z,11Z,14Z-eicosatrienoic acid (5,6-EET), ( \pm 12-hydroxy-5Z,8Z,10E,14Z-eicosatetraenoic acid (12-HETE), ( \pm 15-hydroxy-5Z,8Z,11Z,13E-eicosatetraenoic acid (15-HETE), ( \pm 11-hydroxy-5Z,8Z,12E,14Z-eicosatetraenoic acid (11-HETE), ( \pm 5-hydroxy-6E,8Z,11Z,14Z-eicosatetraenoic acid (5-HETE), 15S-hydroxy-8Z,11Z,13E-eicosatrienoic acid (15-HETrE), 9Shydroxy-11,15-dioxo-2,3,4,5-tetranor-prostan-1,20-dioic acid (tetranor-PGDM), 9-oxo-15S-hydroxy-5Z,10Z,13E-prostatrienoic acid (PGA2), 11-oxo-15S-hydroxy-5Z,9,13E-prostatrienoic acid (PGJ2), 9S-hydroxy-11-oxo-5Z,12E,14E-prostatrienoic acid (15-deoxy- $\delta$-12,14-PGD2), 15S-hydroxy-9-oxo5Z,8(12),13E-prostatrienoic acid (PGB2), ( \pm )5,6-dihydroxy8Z,11Z,14Z,17Z-eicosatetraenoic acid (5,6-DiHETE), 5S,15S-dihydroxy-6E,8Z,10Z,13E-eicosatetraenoic acid (5,15DiHETE), 8S,15S-dihydroxy-5Z,9E,11Z,13E-eicosatetraenoic acid (8,15-DiHETE), 5S,12R-dihydroxy-6Z,8E,10E,14Z-eicosatetraenoic acid (LTB4), 5S,12R-dihydroxy-6E,8E,10E,14Z-eicosatetraenoic acid (6-trans LTB4), ( 1 14,15-dihydroxy5Z,8Z,11Z-eicosatrienoic acid (14,15-DiHETrE), ( \pm 5,6-dihydroxy-8Z,11Z,14Z-eicosatrienoic acid (5,6-DiHETrE), 6-oxo-9S,11R,15S-trihydroxy-2,3-dinor-13E-prostaenoic acid, sodium salt (2,3-dinor-6-keto-PGF1 $\alpha)$, 14S-hydroxy4Z,7Z,10Z,12E,16Z,19Z-docosahexaenoic acid (14-HDoHE), ( \pm 4-hydroxy-5E,7Z,10Z,13Z,16Z,19Z-docosahexaenoic acid (4HDoHE), 9S,15S-dihydroxy-11-oxo-5Z,13E,17Z-prostatrienoic acid (PGD3), 9,15-dioxo-11R-hydroxy-5Z-prostenoic acid (13,14-dihydro-15-keto-PGE2), 9S,11R-epidioxy15S-hydroxy-5Z,13E-prostadienoic acid (PGH2), 9-oxo-11R,15S-dihydroxy-5Z,13E-prostadienoic acid (PGE2), 9S,15S-dihydroxy-11-oxo-5Z,13E-prostadienoic acid (PGD2), 9S,11R-dihydroxy-15-oxo-5Z,13E-prostadienoic acid (15-keto-PGF2 $\alpha)$ 9S,11S-dihydroxy-15-oxo5Z-prostenoic acid (13,14-dihydro-15-keto-PGF2 $\alpha)$, 9S,11R,15S-trihydroxy-5Z,13E-prostadienoic acid (PGF2 $\alpha), \quad 9 S, 11 R, 15 S-t r i h y d r o x y-5 Z, 13 E-p r o s t a d i e n o i c$ acid-cyclo[8S,12R] (8-iso-PGF2 $\alpha), \quad( \pm)$ 19,20-dihydroxy4Z,7Z,10Z,13Z,16Z-docosapentaenoic acid (19,20-DiHDPE), $9 \alpha, 11,15 S$-trihydroxythromba-5Z,13E-dien-1-oic acid (TXB2), 6oxo-9S,11R,15S-trihydroxy-13E-prostenoic acid (6-keto-PGF1 $\alpha$ ), 7S,8R,17S-trihydroxy-4Z,9E,11E,13Z,15E,19Z-docosahexaenoic acid (resolvin D1), and 5S-hydroxy,6R-(Scysteinyl),7E,9E,11Z,14Z-eicosatetraenoic acid (LTE4) standards (see Table S1 for structures) were purchased from Cayman Chemical (Ann Arbor, MI, USA). Fatty acid standards 6Z,9Z,12Z-octadecatrienoic acid ( $\boldsymbol{\gamma}$-linolenic acid), and 9Z,12Z,15Z-octadecatrienoic acid ( $\boldsymbol{\alpha}$-linolenic acid) were purchased from Nu-Chek-Prep (Elysian, MN, USA).

The oxylipins nomenclature and abbreviations follow the LIPID MAPS structure database system [23]. Human plasma samples were obtained from healthy volunteers based on the ethical agreement.

\subsection{Sample preparation}

The stock solution containing 46 eicosanoid and 2 fatty acid standards at the concentration of $500 \mathrm{pg} / \mu \mathrm{L}$ except for tetranorPGDM (2500 pg/ $\mu \mathrm{L}), 2$,3-dinor-6-keto-PGF1 $\alpha$ (2500 pg/ $\mu \mathrm{L})$, PGD3 (2500 pg/ $\mu \mathrm{L}), 5,6$-EET (1000 pg/ $/ \mathrm{L})$, PGH2 (2500 pg/ $\mu \mathrm{L})$, and LTE4 $(5000 \mathrm{pg} / \mu \mathrm{L})$ was prepared in ethanol. For UHPLC measurements, the stock solution was dried under nitrogen at ambient tem- 
perature and redissolved in acetonitrile - water - acetic acid $(45 / 55 / 0.02, \mathrm{v} / \mathrm{v})$ mixture to prepare suitable concentration. In case of UHPSFC analysis, working solutions were prepared in chloroform - ethanol (50/50, v/v) mixture.

The solid phase extraction (SPE) was performed according to the method described by Schebb et al. [24] with minor modifications. Aliquots of $500 \mu \mathrm{L}$ control plasma were diluted with $1.5 \mathrm{~mL}$ $90 \%$ aqueous methanol and vortexed for $10 \mathrm{~s}$. Then the plasma solution was centrifuged (Hettich ${ }^{\circledR}$ EBA 20 centrifuge EBA 20) at $6000 \mathrm{rpm}$ for $10 \mathrm{~min}$ at ambient temperature. The oxylipins were extracted using Strata ${ }^{\mathrm{TM}}-\mathrm{X} 33 \mu \mathrm{m}(200 \mathrm{mg} / 3 \mathrm{~mL})$ polymeric RP cartridges (8B-S100-FBJ-T, Phenomenex, Aschaffenburg, Germany). Cartridges were conditioned with $3 \mathrm{~mL}$ of methanol and then with $3 \mathrm{~mL}$ of water. Next, $2 \mathrm{~mL}$ of samples were loaded on cartridges, with a subsequent wash using $3 \mathrm{~mL}$ of water. Then oxylipins were eluted with $3 \mathrm{~mL}$ of methanol. The eluent was dried under nitrogen at ambient temperature and redissolved in $50 \mu \mathrm{L}$ of the eluent $A$ (acetonitrile - water - acetic acid (45/55/0.02, v/v/v) mixture) for UHPLC or $50 \mu \mathrm{L}$ chloroform - ethanol (50/50, v/v) mixture in case of UHPSFC measurements. Finally, the solution was centrifuged at $6000 \mathrm{rpm}$ for $1 \mathrm{~min}$ prior to transferring into $300 \mu \mathrm{L}$ conical insert.

\subsection{UHPLC/MS and UHPSFC/MS conditions}

UHPLC/MS analyses were performed on a liquid chromatograph Agilent 1290 Infinity series (Agilent Technologies, Waldbronn, Germany) consisting of an Agilent 1290 Infinity LC binary pump, an Agilent 1290 Infinity well plate autosampler and an Agilent 1290 Infinity column thermostat. The UHPLC system was coupled to the Waters Synapt G2-Si quadrupole - ion mobility - time of flight mass spectrometer with ESI (Waters, Milford, MA, USA).

The final UHPLC/MS method for the analysis of oxylipins was as follows: Acquity UPLC BEH C18 column $(150 \times 2.1 \mathrm{~mm}, 1.7 \mu \mathrm{m}$, $130 \AA$, Waters), injection volume $1 \mu \mathrm{L}$, and column temperature $40^{\circ} \mathrm{C}$. The mobile phase A consisted of acetonitrile - water acetic acid (45/55/0.02, v/v/v) mixture, and the eluent B was 2propanol - acetonitrile (50/50, v/v) mixture. The gradient program was the following: $0 \mathrm{~min}-2 \% \mathrm{~B}, 12 \mathrm{~min}-62 \% \mathrm{~B}, 12.1 \mathrm{~min}-99 \% \mathrm{~B}$, $14.5 \mathrm{~min}-99 \% \mathrm{~B}, 15 \mathrm{~min}-2 \% \mathrm{~B}$, and $20 \mathrm{~min}-2 \% \mathrm{~B}$. The flow rate was $0.4 \mathrm{~mL} / \mathrm{min}$ during the analysis (from 0 to $13.5 \mathrm{~min}$ ) and the equilibration before the next injection (from 18.5 to $20 \mathrm{~min}$ ), and $0.65 \mathrm{~mL} / \mathrm{min}$ during the column washing after the analysis (from 13.5 to $18.5 \mathrm{~min}$ ). The injector needle was washed with strong (hexane - 2-propanol - water $(2: 2: 1, \mathrm{v} / \mathrm{v} / \mathrm{v})$ mixture $)$ and weak solvents (A eluent) after each injection.

All UHPSFC/MS experiments were done on an Acquity UPC ${ }^{2}$ system (Waters, Milford, MA, USA) equipped with a binary solvent manager delivery pump, a sample manager maintained at $8^{\circ} \mathrm{C}, 10 \mu \mathrm{L}$ injection loop, a column oven, a backpressure regulator, and a Waters Model 515 pump for delivering make-up solvent. The chromatographic system was coupled to the mass spectrometer via dedicated interface kit (Waters) composed of two T-pieces enabling the backpressure control, and mixing of column effluent with a make-up solvent.

The following UHPSFC columns with the same column dimension $(100 \times 3 \mathrm{~mm}, 1.7 \mu \mathrm{m})$ were tested, i.e., Acquity UPC2 Torus 1-Aminoanthracene (1-AA), Acquity UPC2 Torus 2-Piycolylamine (2-PIC), Acquity UPC2 Torus Diol (1-DIOL), Acquity UPC2 Torus Diethylamine (DEA), and Acquity UPC2 HSS C18 SB $(1.8 \mu \mathrm{m})$ (HSS C18).

The final UHPSFC method for the analysis of oxylipins was as follows: Acquity UPC ${ }^{2}$ Torus 1-Aminoanthracene (1-AA) column $(100 \times 3 \mathrm{~mm}, 1.7 \mu \mathrm{m}$, Waters $)$, active backpressure regulator (ABPR) pressure $1800 \mathrm{psi}$ (124 bar), flow rate $1.7 \mathrm{~mL} / \mathrm{min}$, injection volume $1 \mu \mathrm{L}$, and column temperature $50^{\circ} \mathrm{C}$. The modifier $0.1 \%$ acetic acid in methanol was used as the eluent $\mathrm{B}$. The gradient pro- gram was the following: 0 min - 4\% B, 10 min - 30\% B, 11 min $30 \% \mathrm{~B}, 11.5 \mathrm{~min}-4 \% \mathrm{~B}$, and $15 \mathrm{~min}-4 \% \mathrm{~B}$. The injector needle was washed with hexane - 2-propanol - water (2:2:1, v/v/v) mixture after each injection. Pure methanol was delivered as a make-up eluent at the flow rate of $0.3 \mathrm{~mL} / \mathrm{min}$.

The mass spectrometer was operated in the negative-ion ESI sensitivity mode under the following conditions: mass range of $m / z$ $50-950$, capillary voltage $2.2 \mathrm{kV}$, source temperature $150^{\circ} \mathrm{C}$, sampling cone $20 \mathrm{~V}$, source offset $90 \mathrm{~V}$, drying temperature $500^{\circ} \mathrm{C}$, cone gas flow rate $50 \mathrm{~L} / \mathrm{h}$, desolvation gas flow rate $1000 \mathrm{~L} / \mathrm{h}$, and nebulizer gas pressure 4 bar. Leucine enkephaline was used as a lock mass for all experiments. The data dependent acquisition (DDA) experiments were performed on a transfer cell with the collision energy ramp from 17 to $40 \mathrm{eV}$ with the selection of up to 3 ions. The setting of mass spectrometer was identical for both UHPLC/MS and UHPSFC/MS measurements except for decreased desolvation gas flow rate $(800 \mathrm{~L} / \mathrm{h})$ used for UHPSFC/MS analysis in order to achieve the stable spray.

\subsection{Data processing}

The UHPLC system was controlled with Agilent OpenLAB software. The control of UHPSFC system, MS data acquisition and processing were conducted by MassLynx V4.1 SCN 901 and integrated TargetLynx softwares. Calculations were performed using Microsoft Excel software.

\section{Results and discussion}

A set of 46 oxylipin standards representing 36 eicosanoids, 4 docosanoids, 6 octadecanoids, and 2 fatty acids (see Table S1 for the complete list and structures) was used for the optimization of UHPLC/MS and UHPSFC/MS conditions. The previous occurrence of these standards in human plasma was an important aspect during their selection based on published works [7,12,16-18]. Chromatographic and mass spectrometric parameters were optimized to improve the separation and sensitivity. The final UHPLC/MS and UHPSFC/MS methods were compared, and the former one was applied for profiling of human plasma oxylipins. The MS sensitivity and chromatographic resolution were the main aspects during the optimization due to low natural abundances of oxylipins.

\subsection{Optimization of UHPLC/MS method}

\subsubsection{Column selection and initial conditions}

The Acquity UPLC BEH C18 column was selected for the RP analysis of oxylipins based on the literature $[14,17,25]$. The coelution of oxylipin isomers makes their identification and quantification complicated, therefore longer column $(150 \mathrm{~mm})$ was selected to improve the isomeric separation. The starting conditions were based on the mobile phase consisted of eluent A acetonitrile water - acetic acid (60/40/0.02, v/v/v), eluent B 2-propanol - acetonitrile $(50 / 50, v / v)$, and the gradient program from $0 \mathrm{~min}-0.1 \%$ $\mathrm{B}$ to $6 \mathrm{~min}-55.5 \% \mathrm{~B}$, but too low retention was observed for tetranor-PGDM, 2,3-dinor-6-keto-PGF1 $\alpha$, PGD3, 8-iso-PGF2 $\alpha$, PGF2 $\alpha$, 6-keto-PGF1 $\alpha$, PGE2, PGH2, and TXB2 (retention factors lower than 1.2). Therefore, the amount of water in the eluent $A$ was increased to $55 \%$ water to improve their retention. In parallel, the gradient program was also changed $(0 \mathrm{~min}-0.1 \% \mathrm{~B}, 6 \mathrm{~min}-70.0 \% \mathrm{~B}$, $6.75 \mathrm{~min}-99.0 \% \mathrm{~B}, 7.50 \mathrm{~min}-99 \% \mathrm{~B}, 7.65 \mathrm{~min}-0.1 \% \mathrm{~B}$, and $15 \mathrm{~min}$ $-0.1 \% \mathrm{~B})$.

\subsubsection{Effect of mobile phase composition}

Effects of additives in the eluent $A$ on the retention behavior and MS sensitivity of oxylipins were studied for the following additives: acetic acid (0.02 and $0.05 \%)$, formic acid (0.02 and $0.05 \%)$, 


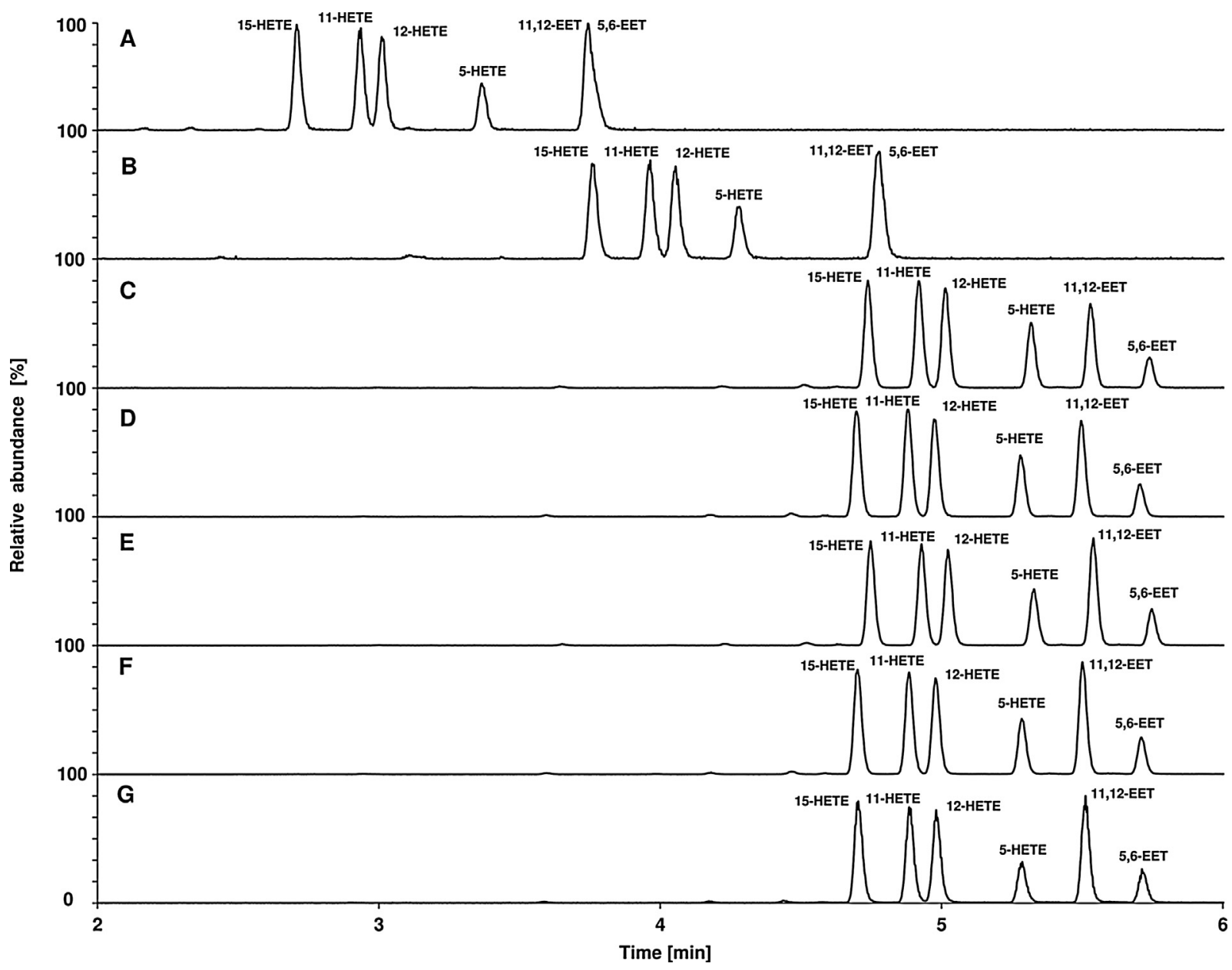

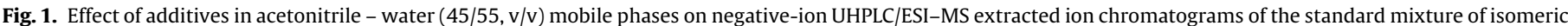

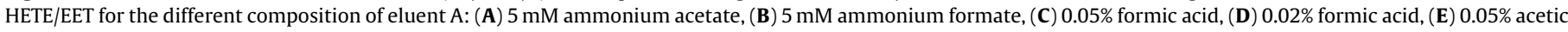

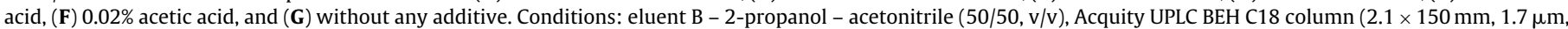
$130 \AA$, Waters), flow rate $0.5 \mathrm{~mL} / \mathrm{min}$, column temperature $40^{\circ} \mathrm{C}$, and gradient program $0 \mathrm{~min}-0.1 \% \mathrm{~B}, 6 \mathrm{~min}-70.0 \% \mathrm{~B}$, and $6.75 \mathrm{~min}-99.0 \% \mathrm{~B}$.

$5 \mathrm{mM}$ ammonium acetate, and $5 \mathrm{mM}$ ammonium formate (Fig. 1). The lowest retention was observed with ammonium acetate, while acetic and formic acids resulted in the highest retention times at $0.05 \%$. The use of acetic and formic acids at the same concentration $(0.02 \%$ or $0.05 \%)$ resulted in different $\mathrm{pH}$ values of the mobile phase, but the retention and resolution of isomers did not change significantly. For all compounds, higher retention times were observed for higher acid content (0.05\%). The comparison of chromatographic resolution for isomers obtained with and without additives show that higher resolution was achieved with acetic acid, formic acid and without any additive, except for $\boldsymbol{\gamma}$-linolenic acid, $\boldsymbol{\alpha}$ linolenic acid, 15-HETE, 11-HETE, 12-HETE, 5-HETE, 11,12-EET, 5,15-DiHETE, 6-trans LTB4, 14-HDHA, and 4-HDHA, where the mobile phases with ammonium acetate provided better results. Peak shapes were not influenced considerably by additives. The serious peak tailing was observed for LTE4 in the absence of any additive and in ammonium acetate or ammonium formate containing mobile phases. The peak shape was improved at higher acid concentrations, which can be explained by suppressed zwitterion formation of cysteinyl side chain (isoelectric point at $\mathrm{pH}$ 5.07) in more acidic environment [26]. The oxalic acid treatment of stationary phase using the injection of $20 \mu \mathrm{L}$ of $10 \mathrm{mM}$ oxalic acid two times [27] had also a substantial effect on the improvement of peak shape of LTE4.

Fig. 2 demonstrates the dependence of negative-ion ESI response on the type and concentration of additives. Acetic acid

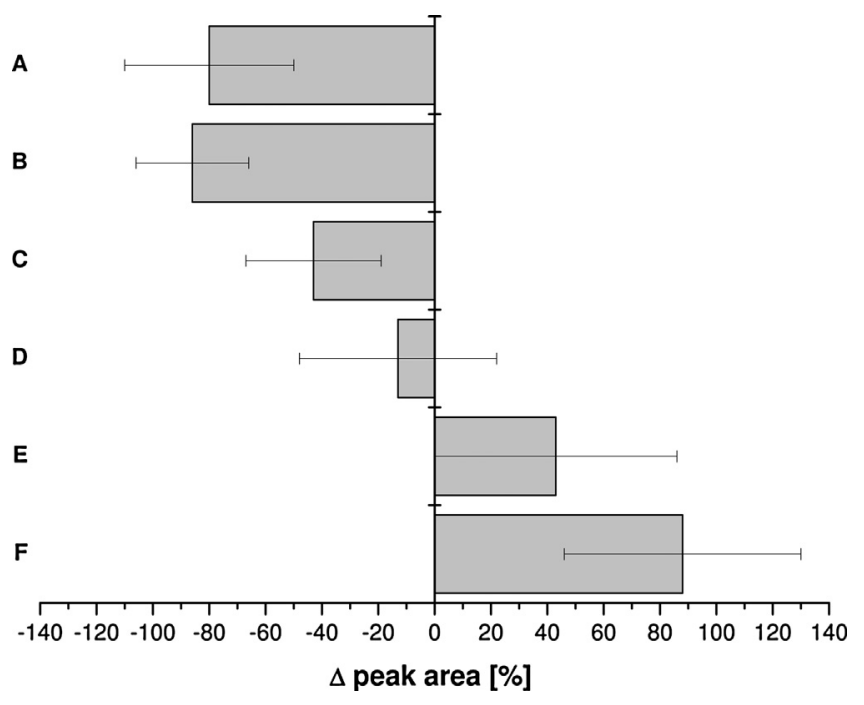

Fig. 2. Dependencies of peak areas of oxylipins standards in negative-ion UHPLC/ESI-MS on the composition of eluent A containing various additives in comparison to conditions without any additives: (A) $5 \mathrm{mM}$ ammonium acetate, (B) $5 \mathrm{mM}$ ammonium formate, (C) $0.05 \%$ formic acid, (D) $0.02 \%$ formic acid, (E) $0.05 \%$ acetic acid, and (F) $0.02 \%$ acetic acid. Other conditions are identical as for Fig. 1. 
Table 1

Average relative changes in UHPLC for retention times $\left(\Delta t_{R}\right)$, peak widths, peak areas, and resolution $\left(R_{s}\right)$ for increased flow rates of 0.4 and $0.5 \mathrm{~mL} / \mathrm{min}$ in comparison to the initial value of $0.3 \mathrm{~mL} / \mathrm{min}$.

\begin{tabular}{lllll}
\hline Flow rate $[\mathrm{mL} / \mathrm{min}]$ & $\Delta \mathrm{t}_{\mathrm{R}}$ & $\Delta$ peak width & $\Delta$ peak area & $\Delta R_{s}$ \\
\hline 0.4 & $-33 \%$ & $-21 \%$ & $-31 \%$ & $-6 \%$ \\
0.5 & $-54 \%$ & $-33 \%$ & $-51 \%$ & $-14 \%$ \\
\hline
\end{tabular}

enhanced the ionization compared to the mobile phase without any additive (ca. $90 \%$ on the average for $0.02 \%$ and $40 \%$ for $0.05 \%$ of acetic acid). The use of formic acid caused the signal suppression (ca. $15 \%$ for $0.02 \%$ and $45 \%$ for $0.05 \%$ of formic acid). The addition of ammonium acetate or formate resulted in the drastic loss of sensitivity. $0.02 \%$ of acetic acid was selected as optimal for the further optimization of other parameters.

The retention and selectivity of the separation can be tuned by the composition of the organic solvent B. Four types of eluents B were investigated, such as 2-propanol - acetonitrile (50/50, v/v), 2-propanol - acetonitrile (10/90, v/v), acetonitrile, and methanol. The concentration of 2-propanol exhibited only a slight effect on retention, resolution, and sensitivity. The highest retention was observed for methanol, but it negatively influenced the separation, especially in case of $\boldsymbol{\gamma}$-linolenic acid, $\boldsymbol{\alpha}$-linolenic acid, 13-HODE, 9-HODE, and all HETE. Finally, 2-propanol - acetonitrile (50/50, v/v) was selected, because this was the most efficient in the removal of plasma phospholipid contaminants (in spite of SPE sample treatment) from the column during the washing step of gradient program.

\subsubsection{Effect of flow rate}

The increased flow rate reduces the analysis time, but on the other hand it also influences the resolution and sensitivity. Fig. 3 demonstrates the effect of flow rate in the range of $0.3-0.5 \mathrm{~mL} / \mathrm{min}$ for isomers 15-HETE, 11-HETE, 12-HETE, 5-HETE, 11,12-EET, and 5,6-EET. Gradients for different flow rates are recalculated based on the ratio of squared column internal diameters. Average retention times and peak widths were decreased for all compounds, when the flow rate increased (Table 1 ), but it also reduced peak areas. The moderate reduction of resolution was observed with increased flow rates for isomers. $0.4 \mathrm{~mL} / \mathrm{min}$ is chosen in the final method as a compromise among the short analysis time, high selectivity, and ESI response. The oxylipin and fatty acid standards eluted in the range of $1-12 \mathrm{~min}$ (Fig. 4 and Table 2). The flow rate gradient program was used during the washing and equilibration cycle to reduce the total run time.

\subsection{Development of UHPSFC/MS method}

\subsubsection{Column selection}

Five UHPSFC columns with the same column dimension $(100 \times 3 \mathrm{~mm}, 1.7 \mu \mathrm{m})$ and different chemistries were used in our column screening, i.e., 1-AA, 2-PIC, 1-DIOL, DEA, and HSS $\mathrm{C} 18$, in order to find the optimal stationary phase for the anal-

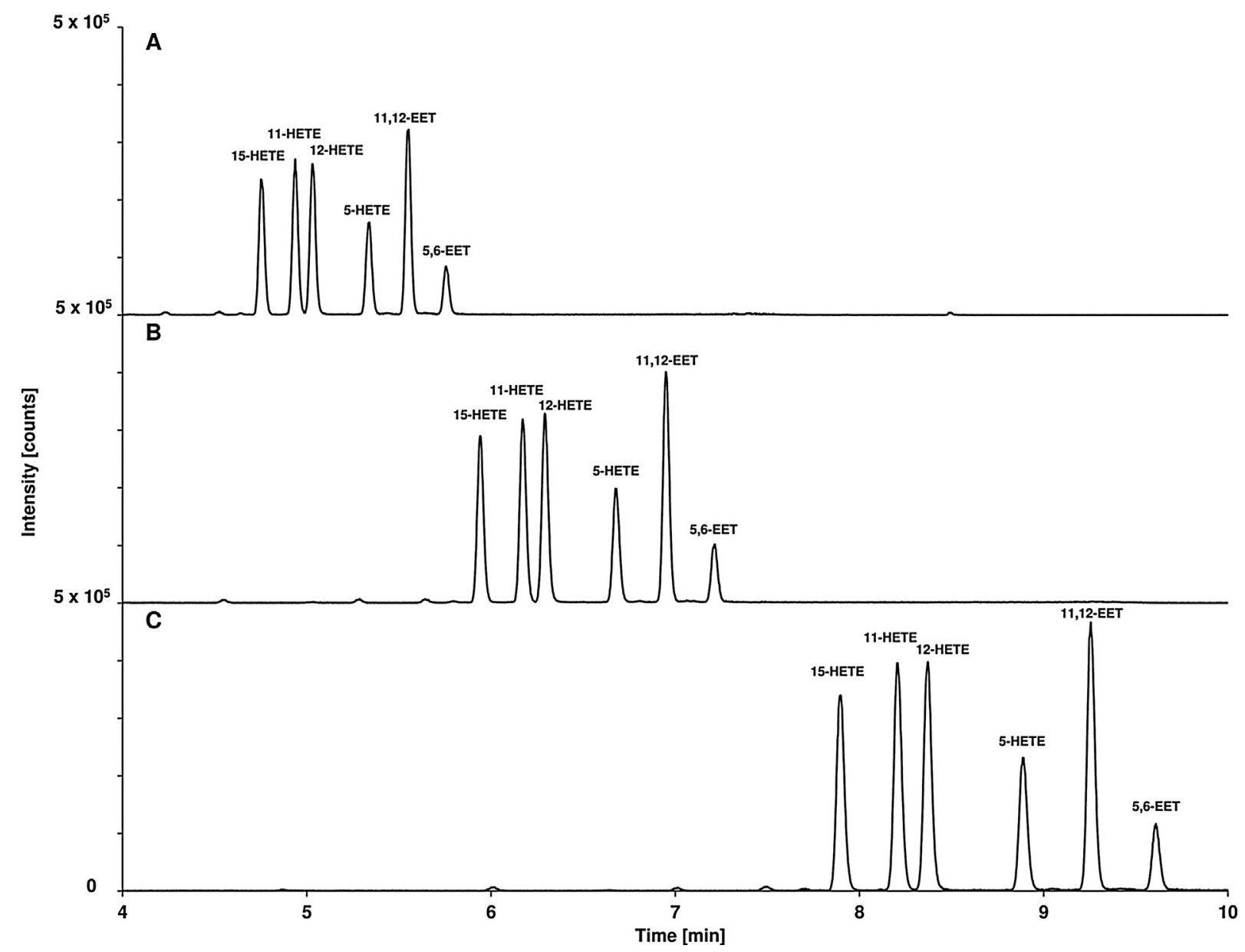

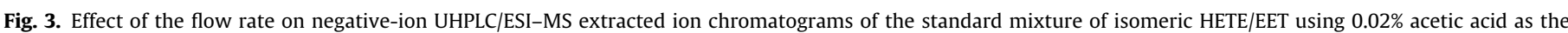

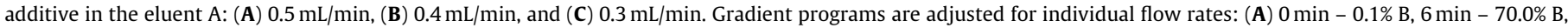

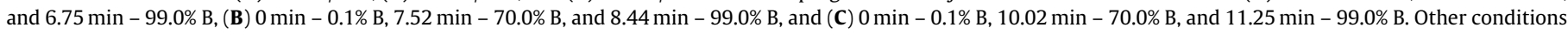
are identical as for Fig. 1. 
Table 2

Chromatographic and mass spectrometric data for oxylipin and fatty acid standards in the final UHPLC/MS method.

\begin{tabular}{|c|c|c|c|c|c|c|}
\hline Abbreviation & Subclass & Isomeric group & Theoretical $m / z$ of $[\mathrm{M}-\mathrm{H}]^{-}$ & $t_{R}$ in UHPLC/MS [min] & $\mathrm{RS}_{S}$ & Indentified in plasma \\
\hline tetranor-PGDM & Prostaglandins & - & 327.1449 & 0.96 & & No \\
\hline 6-keto-PGF1 $\alpha$ & Prostaglandins & 1 & 369.2283 & 1.24 & 1.25 & No \\
\hline TXB2 & Thromboxanes & & & 1.53 & & Yes \\
\hline 8 -iso-PGF2 $\alpha$ & Prostaglandins & 2 & 353.2334 & 1.52 & 1.53 & Yes \\
\hline PGF2 $\alpha$ & & & & 1.73 & 4.96 & Yes \\
\hline 13,14-dihydro-15-keto-PGF2 $\alpha$ & & & & 2.43 & & No \\
\hline 2,3-dinor-6-keto-PGF1 $\alpha$ & Prostaglandins & - & 341.1970 & 1.71 & & No \\
\hline PGD3 & Prostaglandins & - & 349.2021 & 1.71 & & No \\
\hline PGH2 & Prostaglandins & 3 & 351.2177 & 1.88 & & No \\
\hline PGE2 & & & & 1.88 & 1.34 & Yes \\
\hline 15-keto-PGF2 $\alpha$ & & & & 2.03 & 0.68 & No \\
\hline PGD2 & & & & 2.08 & 3.35 & Yes \\
\hline 13,14-dihydro-15-keto-PGE2 & & & & 2.48 & & No \\
\hline Resolvin D1 & Docosanoids & - & 375.2177 & 2.37 & & No \\
\hline PGA2 & Prostaglandins & 4 & 333.2071 & 3.15 & 0.96 & No \\
\hline PGJ2 & & & & 3.33 & & No \\
\hline PGB2 & & & & 3.33 & 7.51 & Yes \\
\hline 15-deoxy- $\delta-12,14-P G D 2$ & & & & 4.51 & & No \\
\hline 8,15-DiHETE & Hydroxy/hydroperoxyeicosatetraenoic & 5 & 335.2228 & 3.85 & 2.15 & Yes \\
\hline 5,15-DiHETE & acids & & & 4.14 & 0.71 & Yes \\
\hline 6-trans LTB4 & Leukotrienes & & & 4.22 & 1.55 & Yes \\
\hline LTB4 & & & & 4.48 & 6.33 & No \\
\hline 5,6-DiHETE & Hydroxy/hydroperoxyeicosatetraenoic acids & & & 5.54 & & Yes \\
\hline 12,13-DiHOME & Other octadecanoids & - & 313.2384 & 4.75 & & Yes \\
\hline 19,20-DiHDPE & Docosanoids & - & 361.2384 & 5.33 & & Yes \\
\hline tetranor-12-HETE & Hydroxy fatty acids & - & 265.1809 & 5.34 & & No \\
\hline 14,15-DiHETrE & Hydroxy/hydroperoxyeicosatrienoic & 6 & 337.2384 & 5.40 & 8.46 & Yes \\
\hline 5,6-DiHETrE & acids & & & 7.18 & & Yes \\
\hline 12-HHTrE & Hydroxy/hydroperoxyeicosatrienoic acids & - & 279.1966 & 5.50 & & Yes \\
\hline LTE4 & Leukotrienes & - & 438.2320 & 5.60 & & No \\
\hline 13-HOTrE & Other octadecanoids & 7 & 293.2122 & 6.34 & 7.77 & Yes \\
\hline 13-OxOODE & & & & 7.83 & 1.72 & Yes \\
\hline 9-OxoODE & & & & 8.22 & & Yes \\
\hline 15-HEPE & Hydroxy/hydroperoxyeicosapentaenoic acids & 8 & 317.2122 & 6.70 & 4.94 & Yes \\
\hline 5-HEPE & acids & & & 7.55 & & Yes \\
\hline 13-HODE & Other octadecanoids & 9 & 295.2279 & 7.56 & 1.10 & Yes \\
\hline 9-HODE & & & & 7.73 & & Yes \\
\hline 15-HETE & Hydroxy/hydroperoxyeicosatetraenoic & 10 & 319.2279 & 7.85 & 2.23 & Yes \\
\hline 11-HETE & acids & & & 8.26 & 1.20 & Yes \\
\hline 12-HETE & & & & 8.48 & 3.39 & Yes \\
\hline 5-HETE & & & & 9.13 & 3.05 & Yes \\
\hline 11,12-EET & Epoxyeicosatrienoic & & & 9.72 & 2.32 & No \\
\hline 5,6-EET & acids & & & 10.16 & & No \\
\hline 14-HDoHE & Docosanoids & 11 & 343.2279 & 8.17 & 6.26 & Yes \\
\hline 4-HDoHE & & & & 9.34 & & Yes \\
\hline 15-HETrE & Hydroxy/hydroperoxyeicosatrienoic acids & - & 321.2435 & 8.68 & & Yes \\
\hline$\alpha$-Linolenic acids & Unsaturated fatty acids & 12 & 277.2173 & 11.70 & 0.91 & Yes \\
\hline$\gamma$-Linolenic acids & & & & 11.90 & & Yes \\
\hline
\end{tabular}




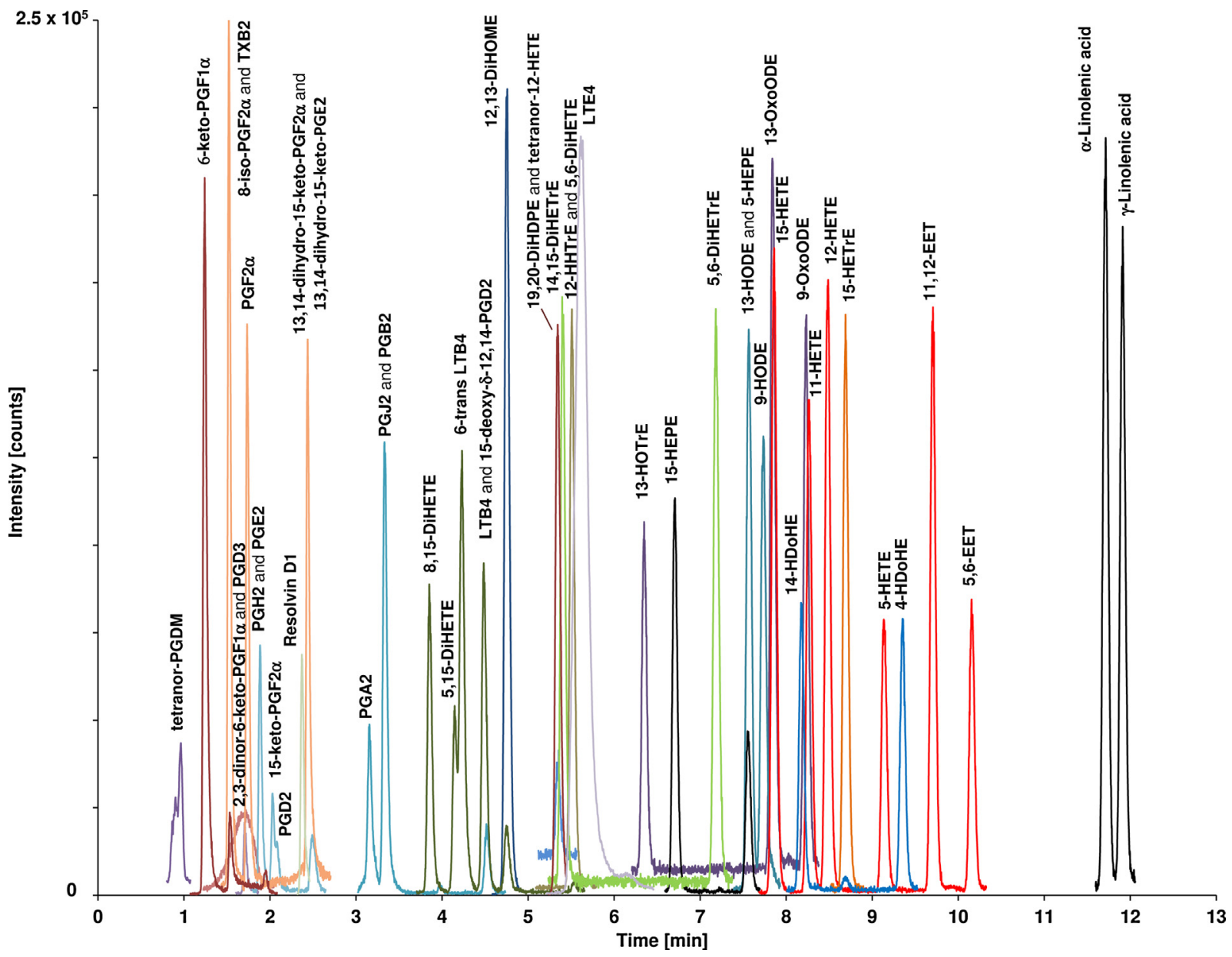

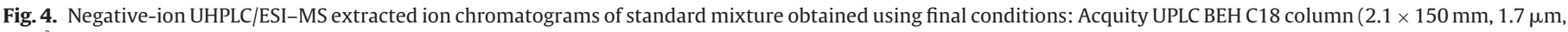

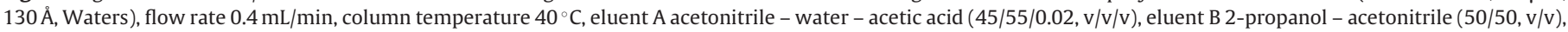
and gradient program $0 \mathrm{~min}-2 \% \mathrm{~B}, 12 \mathrm{~min}-62 \% \mathrm{~B}$, and $12.1 \mathrm{~min}-99 \% \mathrm{~B}$. Details are in the Experimental.

ysis of oxylipins. The following parameters were chosen for the initial optimization: eluent B $10 \mathrm{mM}$ ammonium acetate in methanol, methanol as the make-up eluent, ABPR pressure $1800 \mathrm{psi}$ (124.1 bar), flow rate $1.0 \mathrm{~mL} / \mathrm{min}$, injection volume $1 \mu \mathrm{L}$, column temperature $50^{\circ} \mathrm{C}$, and the gradient program $0 \mathrm{~min}-1 \% \mathrm{~B}, 5 \mathrm{~min}$ - 50\% B, $12 \mathrm{~min}-50 \%$ B, and $13 \mathrm{~min}-1 \% \mathrm{~B}$. First, the retention behavior of selected standards representing different types of isomerism was studied (PGA2, 15-deoxy- $\boldsymbol{\delta}-\mathbf{1 2 , 1 4 - P G D 2 , ~ P G B 2 , ~}$ PGD2, PGE2, PGF2 $\alpha$, 8-iso-PGF2 $\alpha$, TXB2, and 6-keto-PGF1 $\alpha$ ). For all compounds, the lowest retention was observed on HSS C18 column compared with other stationary phases, moreover PGF2 $\alpha$, 8-iso-PGF2 $\alpha$, PGD2, and PGE2 are not separated. The obtained low retention and poor selectivity indicated the importance of electrostatic attraction between the carboxylate group from analytes and the basic functionality in the stationary phase, which is not occurring at all or only with mild effects on HSS C18 stationary phase due to the lack of additional ionizable functional groups beside residual silanols $\left(\mathrm{pK}_{\mathrm{a}}=7-8\right)[28,29]$. DEA had the most basic functionality (calculated basic $\mathrm{pK}_{\mathrm{a}}=9.5$ ), providing strong electrostatic interactions with deprotonated carboxyl groups of oxylipins, thus resulting in the highest retention times for all analytes, and generally accompanied by highest peak width, which resulted in the lower resolution. HSS C18 and DEA columns were excluded from further optimization based on above mentioned observations. Comparable retention and selectivity were observed on the remaining three stationary phases, therefore those were further tested. 1 -DIOL provided lower retention of oxylipins compared to the basic stationary phases. Lower retention times were observed for all analytes on 1-AA relative to 2-PIC column [29]. Fig. 5 demonstrated that 1-AA stationary phase provided a superior chromatographic resolution for 11,12-EET, 12-HETE, 15-HETE, 11-HETE, and 5HETE isomers over 1-DIOL and 2-PIC columns. For $\gamma$-linolenic acid and $\alpha$-linolenic acid, the partial separation was obtained only on 1-AA stationary phase. The bulky anthracene ring may improve the recognition process of isomers in at least two ways, such as the formation of additional hydrophobic interactions and steric hindrance. Finally, 1-AA column was selected for the development of UHPSFC/MS method considering the chromatographic data obtained for HETE, which exhibit numerous biologically important isomers in plasma.

\subsubsection{Effect of flow rate}

One of the main benefits of UHPSFC is the feasibility of using high flow rate $[30,19]$, therefore $1.0,1.5,1.7,2.0$, and $2.3 \mathrm{~mL} / \mathrm{min}$ flow rates were tested. The increased the flow rate from $1.0 \mathrm{~mL} / \mathrm{min}$ resulted in the following average reductions of retention times: $36 \%$ at $1.5 \mathrm{~mL} / \mathrm{min}, 44 \%$ at $1.7 \mathrm{~mL} / \mathrm{min}, 53 \%$ at $2.0 \mathrm{~mL} / \mathrm{min}$, and $60 \%$ at $2.3 \mathrm{~mL} / \mathrm{min}$. The average resolution of isomers changed only moderately, and the maximum was found at $1.5 \mathrm{~mL} / \mathrm{min}$. The resolution was only slightly lower at $1.7 \mathrm{~mL} / \mathrm{min}$. The increase of the flow rate from 1.5 to $1.7 \mathrm{~mL} / \mathrm{min}$ decreased peak widths, especially for tetranor-PGDM eluting at the beginning. The decrease of observed peak areas was very small (only $3 \%$ ). Therefore, $1.7 \mathrm{~mL} / \mathrm{min}$ was used in the final method. 


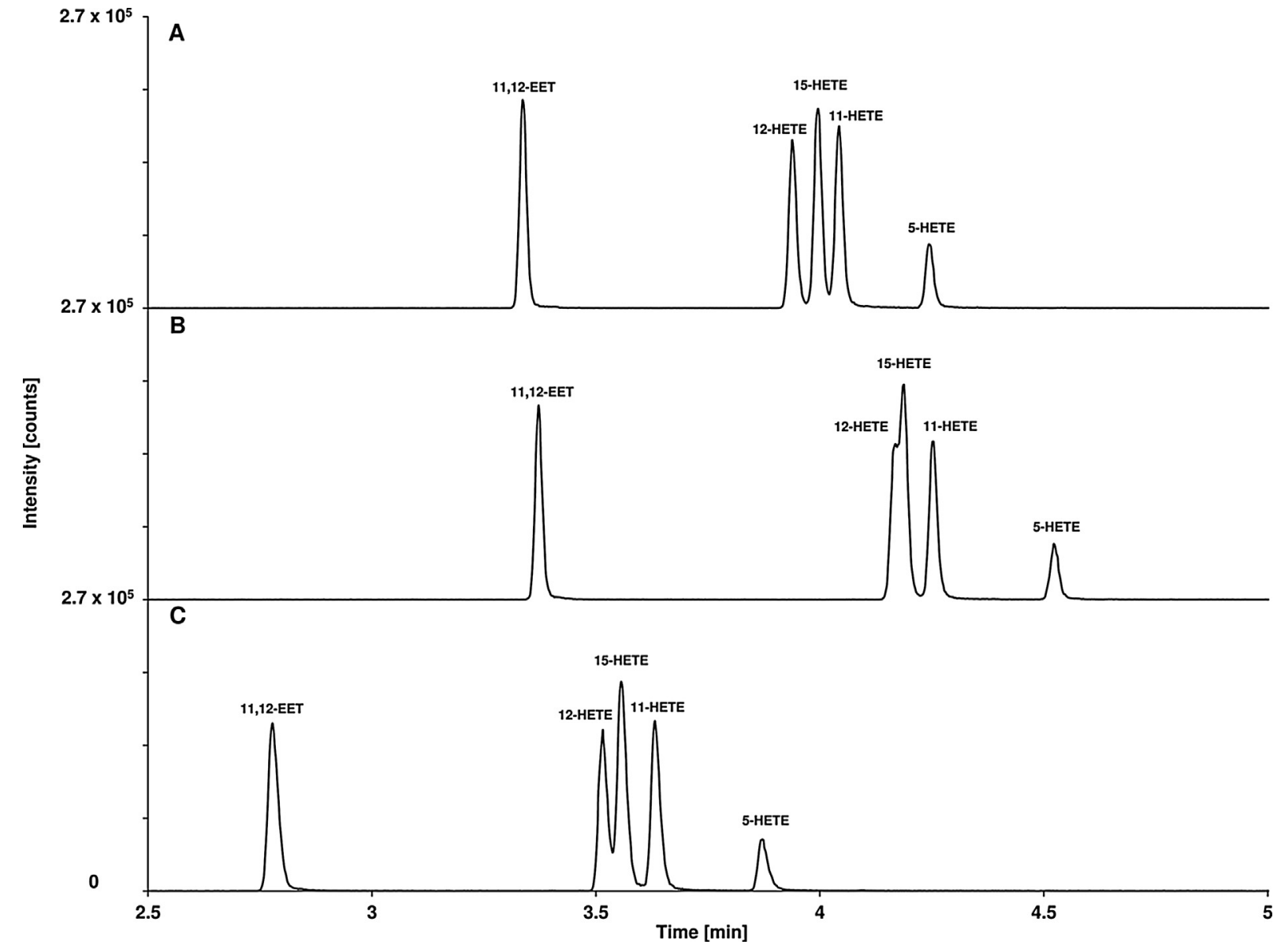

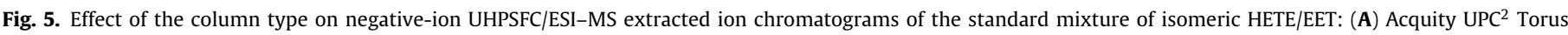

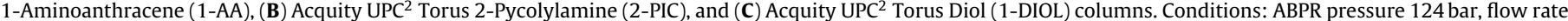
$1 \mathrm{~mL} / \mathrm{min}$, column temperature $50^{\circ} \mathrm{C}$, modifier $10 \mathrm{mM}$ ammonium acetate, gradient program $0 \mathrm{~min}-1 \% \mathrm{~B}, 10 \mathrm{~min}-50 \% \mathrm{~B}$, and $\mathrm{make}$-up pump flow rate $0.2 \mathrm{~mL} / \mathrm{min}$.

The effect of flow rate of make-up eluent on the sensitivity was also studied, and the trend of average signal reduction with increasing flow rate from $0.15 \mathrm{~mL} / \mathrm{min}$ was the following: $10 \%$ at $0.2 \mathrm{~mL} / \mathrm{min}, 13 \%$ at $0.25 \mathrm{~mL} / \mathrm{min}$ and $20 \%$ at $0.30 \mathrm{~mL} / \mathrm{min}$. Despite the lower sensitivity at higher flow rate, $0.30 \mathrm{~mL} / \mathrm{min}$ is essential to achieve stable spray in ESI-MS, especially at the beginning of analysis with low modifier content.

\subsubsection{Effect of temperature and backpressure}

The selectivity and mainly the retention in UHPSFC can be influenced by the density of mobile phase through the regulation of temperature and backpressure [31]. In order to study the effect of temperature on the chromatographic behavior of oxylipins, 40 , 50 , and $60^{\circ} \mathrm{C}$ were selected at 124 bar backpressure. For all compounds, higher retention times are observed at higher temperature, while the separation of isomers was not influenced significantly. The effect of temperature on the change of retention was reduced by increasing the content of organic modifier in the mobile phase during the gradient program (Fig. S1). The distribution of data correlates with graphs obtained for diffusion coefficients of anthocyanins dependence on the methanol content in the mobile phase [32]. This retention behavior might be explained by decreased diffusion coefficients of oxylipins with increased organic solvent content and probably by the transition from supercritical to subcritical state in the range of $12-16 \%$ methanol $[32,33]$. The similar chromatographic behavior was found with decreased backpressure from 138 to 103 bar. The column temperature of $50{ }^{\circ} \mathrm{C}$ and the backpressure of 124 bar were selected in the final method.

\subsubsection{Effect of gradient steepness}

The retention and selectivity in UHPSFC can be influenced by the gradient steepness similarly as for other chromatographic techniques [31]. $30 \%$ of organic modifier provides sufficient elution strength even for the elution of more retained polar standards, for example tetranor-PGDM and 2,3-dinor-6-keto-PGF1 $\alpha$. Thus, the range from $4 \%$ to $30 \%$ of the eluent B was used for the initial optimization step. In order to investigate the effect of gradient steepness on the chromatographic retention, a study was carried out with the gradient slope of $2.6,5.2$, and $10.4 \%$ of $B / \mathrm{min}$. Retention times increased with decreased gradient steepness. This increase was $36 \%$ at $5.2 \%$ of $\mathrm{B} / \mathrm{min}$ and $86 \%$ at $2.6 \%$ of $\mathrm{B} / \mathrm{min}$ on the average. For the separation of isomers, a similar trend was found in the increase of resolution (57\% and $152 \%$ ) with the decrease of the gradient steepness.

\subsubsection{Selection of organic co-solvent and mobile phase additives}

The polarity of supercritical carbon dioxide is similar to hexane, thus the use of polar organic modifier in the mobile phase is essential for the elution of more polar compounds [19]. Methanol is the most common organic modifier in UHPSFC owing to its physical and chemical properties, such as its high elution strength, and the complete miscibility with supercritical fluid carbon dioxide at given temperature and pressure ranges. Moreover, the mobile phase additives (e.g., ammonium acetate or formate) are well soluble in methanol at typically used concentrations [31]. Methanol with $10 \mathrm{mM}$ of ammonium acetate was used as the organic modifier in the initial stage of optimization. The effect of other organic 


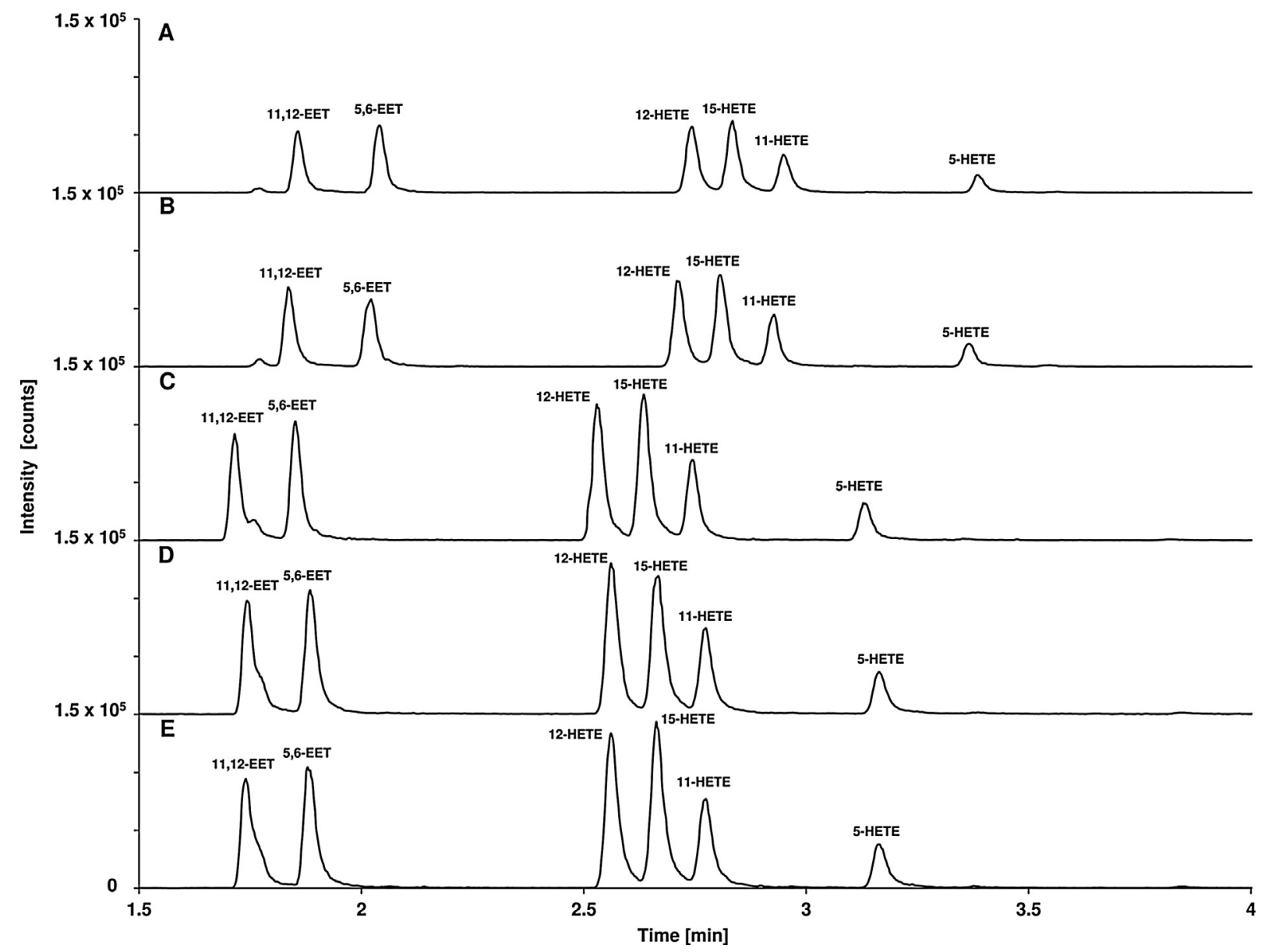

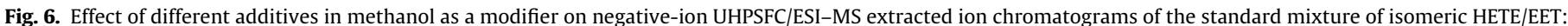

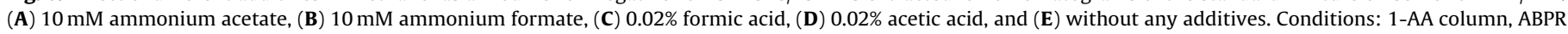

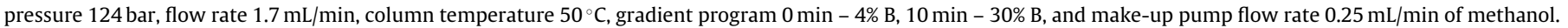

modifiers in methanol was tested for $10 \%$ or $20 \%$ of acetonitrile or 2-propanol in methanol. For all compounds, the addition of either acetonitrile or 2-propanol increased the retention moderately, while no influence on the separation of isomers was detected, therefore only methanol is used in further optimization steps.

The application of polar additives, such as acid, base, neutral salt or buffer in the mobile phase can influence the retention, selectivity and sensitivity through different ways depending on properties (mainly acid - base) of analyte, types of packed stationary phase and the detection mode [29,19,31]. Considering basic properties of 1-AA column and the detection of acidic oxylipins by negative-ion mode ESI-MS, the effects of acetic acid, formic acid, ammonium acetate, and ammonium formate on the chromatographic behavior and the sensitivity were investigated and compared with the mobile phase without any additive. The nature of additives showed only moderate effects on the retention, while the sensitivity was influenced considerably. Fig. 6 reveals that the use of different mobile phase additives for 1,12-EET, 12-HETE, 15-HETE, 11-HETE, and 5-HETE isomers, slightly lower retention was observed for formic acid, while higher retention times were obtained when using salts, especially with ammonium acetate. Generally, increased retention and slightly improved resolution were obtained for increased concentration of ammonium acetate $(1,5,10,20$, and $30 \mathrm{mM})$. However, the increase of acetic acid content $(0.01,0.02,0.05,0.1$, and $0.2 \%)$ did not influence the retention and selectivity. The effect of additives on peak shape was negligible.

The concentration of acetic acid in the range of $0-0.2 \%$ does not show any visible effect on the retention. Fig. 6 shows the role of additives in the MS response. The use of ammonium acetate provided the lowest responses, as general trend for all compounds (47\%), while the highest peak areas were obtained without any additives (i.e., 100\%) in methanol. Slightly higher response was found for ammonium formate (61\%). The reduction of signal was more considerable for increased concentration of ammonium acetate, for example the mean intensity was reduced to approximately $50 \%$ with the change of concentration from 1 to $30 \mathrm{mM}$. Acetic acid (96\%) provided a better sensitivity than formic acid $(85 \%)$. The mean peak area showed a maximum at $0.1 \%$ of acetic acid within the tested range from 0 to $0.2 \%$. The use of buffers in the mobile phase slightly improved the separation of isomers, but on the other hand it resulted in a serious loss of sensitivity, therefore $0.1 \%$ acetic acid was selected as the mobile phase additive.

The effect of water content $(1,2$, and $5 \%)$ in the mobile phase containing $0.1 \%$ acetic acid on the retention and selectivity was studied. A slight improvement of peak shapes was achieved with $5 \%$ of water, but the ESI response was dramatically reduced by $65 \%$. The similar signal reduction was observed for $1 \%$ water in methanol used as a make-up solvent, therefore water was not used either in the mobile phase or make-up solvent in further experiments.

\subsubsection{Effect of sample solvent and injected volume}

The type of solvent and injected volume can influence the peak shape and the retention time as well. Hexane and heptane are the best choice for dissolution solvent due to the similar polarity with supercritical carbon dioxide [19]. Hexane and chloroform were selected as commonly used nonpolar solvents in lipidomics. 


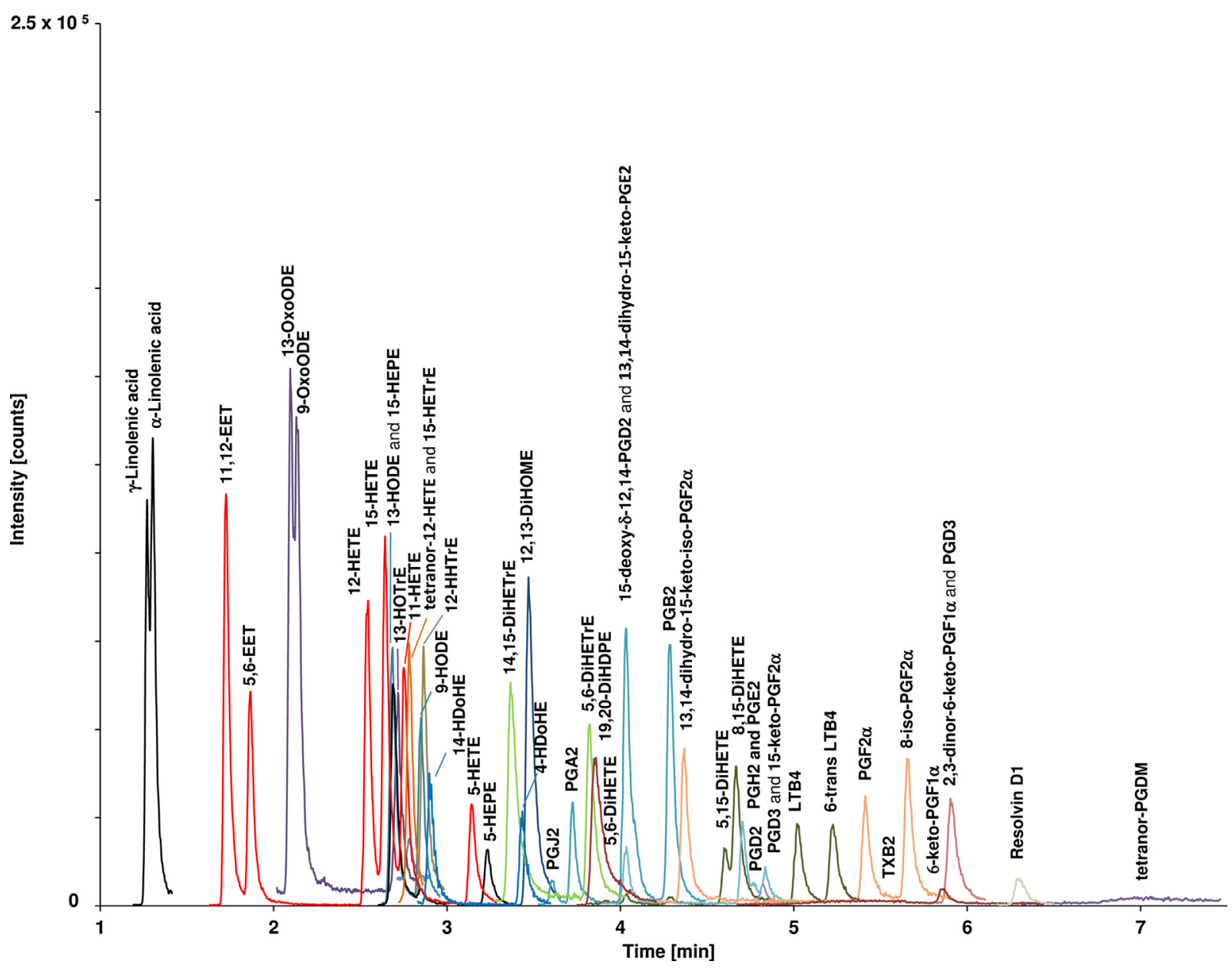

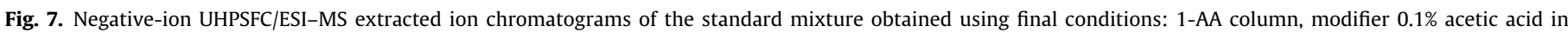

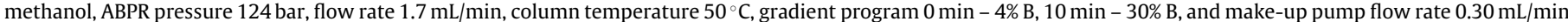
of methanol. Details are in the Experimental.

However, the addition of polar organic solvent into chloroform was necessary for more polar standards. Thus, the effect of methanol and ethanol content and type of nonpolar solvents was investigated on chromatographic results. No significant changes were observed for retention times. The use of hexane and chloroform with $50 \%$ ethanol content showed that chloroform resulted in narrower peak widths. Concerning contribution of polar organic solvent (50\%) in chloroform, the addition of ethanol resulted in better peak shape than methanol, additionally better separation occurred in case of low retained linolenic acid isomers. Interestingly, higher chloroform content did not provide any improvement in peak shapes. The selection of chloroform - ethanol $(50 / 50, v / v)$ was the best compromise considering peak profiles, proper polarity, and low volatility. The injected volume may have the main effect on the peak distortion [19], therefore measurements were carried out for the injection volumes of $1,2,3$, and $5 \mu \mathrm{L}$. The increase of injection volume resulted in no change in the retention, but it decreased the chromatographic resolution, as expected. This trend was more evident for less retained compounds. Thus, the injected volume was limited to $1 \mu \mathrm{L}$.

The final UHPSFC/MS method provided baseline separation for 20 oxylipin standards within 7 min (Fig. 7 and Table 3).

\subsection{Comparison of UHPLC/MS and UHPSFC/MS}

Fig. 8 demonstrated different retention mechanisms with a low degree of orthogonality $\left(r^{2}=0.8\right)$ between UHPLC/MS and
UHPSFC/MS methods. Generally, the increase of the compound hydrophobicity resulted in higher retention in RP-UHPLC, while the opposite trend was observed in UHPSFC. A different elution order between UHPLC and UHPSFC was observed for isomeric groups 1, 2, $3,4,5,7,10$, and 12. For $\gamma$-linolenic acid, $\alpha$-linolenic acid, 11,12EET, and 5,6-EET, the highest retention was observed in UHPLC, while lowest retention times were obtained in UHPSFC. Resolvin D1, 2,3-dinor-6-keto-PGF1 $\alpha$, TXB2, and 6-keto-PGF1 $\alpha$ with 3 hydroxyl groups provided highest retention times in UHPSFC. For TXB2, broad (in UHPSFC) and tailing (in UHPLC) elution profiles may be associated with the interconversion between hemiacetal isomers [34]. The presence of additional carboxyl group in more polar analyte tetranor-PGDM caused the tailing peak with increased retention in UHPSFC. The contribution of polar cysteinyl side chain of LTE4 may contribute to the peak shape distortion. This unfavorable effect was more pronounced in UHPSFC, where it disabled the detection of LTE4 due to extremely tailing peaks. The degree of unsaturation also influenced the retention, mainly in UHPLC. Table 2 reveals that for 15-HETrE, 15-HETE, and 15-HEPE, the reduction in retention times correlates with the increase in the number of double bonds.

Tables 2 and 3 show that UHPLC generally provides better separation of isomers than UHPSFC method except for positional isomeric groups of $\mathbf{2}, \mathbf{4}, \mathbf{5}$, and $\mathbf{9}$. Higher retention and resolution were observed for positional isomers PGJ2, PGA2, and PGB2 in UHPSFC. It indicates that the position of the carbonyl group at C9 was more favorable for dipole-dipole interaction than at $\mathrm{C} 11$, thus 
Table 3

Chromatographic and mass spectrometric data for oxylipin and fatty acid standards in the final UHPSFC/MS method.

\begin{tabular}{|c|c|c|c|c|c|c|}
\hline Abbreviation & Subclass & Isomeric group & Theoretical $m / z$ of $[\mathrm{M}-\mathrm{H}]^{-}$ & $t_{R}$ in UHPSFC/MS [min] & $\mathrm{R}_{\mathrm{S}}$ & Indentified in plasma \\
\hline tetranor-PGDM & Prostaglandins & - & 327.1449 & 7.03 & & No \\
\hline TXB2 & Thromboxanes & 1 & 369.2283 & 5.36 & 1.02 & Yes \\
\hline 6-keto-PGF1 $\alpha$ & Prostaglandins & & & 5.86 & & No \\
\hline 13,14-dihydro-15-keto-PGF2 $\alpha$ & Prostaglandins & 2 & 353.2333 & 4.37 & 7.23 & No \\
\hline PGF2 $\alpha$ & & & & 5.41 & 1.75 & Yes \\
\hline 8-iso-PGF2 $\alpha$ & & & & 5.66 & & Yes \\
\hline 2,3-dinor-6-keto-PGF1 $\alpha$ & Prostaglandins & - & 341.1970 & 5.90 & & No \\
\hline PGD3 & Prostaglandins & - & 349.2021 & 4.82 & & No \\
\hline 13,14-dihydro-15-keto-PGE2 & Prostaglandins & 3 & 351.2177 & 4.03 & 4.81 & No \\
\hline PGH2 & & & & 4.71 & & No \\
\hline PGE2 & & & & 4.71 & 0.68 & Yes \\
\hline PGD2 & & & & 4.77 & 0.78 & Yes \\
\hline 15-keto-PGF2a & & & & 4.83 & & No \\
\hline Resolvin D1 & Docosanoids & - & 375.2177 & 6.30 & & No \\
\hline PGJ2 & Prostaglandins & 4 & 333.2071 & 3.61 & 1.02 & No \\
\hline PGA2 & & & & 3.73 & 3.07 & No \\
\hline 15-deoxy- $\delta-12,14-P G D 2$ & & & & 4.08 & 2.10 & No \\
\hline PGB2 & & & & 4.28 & & Yes \\
\hline 5,6-DiHETE & Hydroxy/hydroperoxyeicosatetraenoic & 5 & 335.2228 & 3.91 & 9.12 & Yes \\
\hline 5,15-DiHETE & acids & & & 4.60 & 0.73 & Yes \\
\hline 8,15-DiHETE & & & & 4.67 & 2.40 & Yes \\
\hline LTB4 & Leukotrienes & & & 5.02 & 1.32 & No \\
\hline 6-trans LTB4 & & & & 5.22 & & Yes \\
\hline 12,13-DiHOME & Other octadecanoids & - & 313.2384 & 3.47 & & Yes \\
\hline 19,20-DiHDPE & Docosanoids & - & 361.2384 & 3.86 & & Yes \\
\hline tetranor-12-HETE & Hydroxy fatty acids & - & 265.1809 & 2.78 & & No \\
\hline 14,15-DiHETrE & Hydroxy/hydroperoxyeicosatrienoic & 6 & 337.2384 & 3.37 & 2.60 & Yes \\
\hline 5,6-DiHETrE & acids & & & 3.82 & & Yes \\
\hline 12-HHTrE & Hydroxy/hydroperoxyeicosatrienoic acids & - & 279.1966 & 2.87 & & Yes \\
\hline LTE4 & Leukotrienes & - & 438.2320 & n.d. & & No \\
\hline 13-OxoODE & Other octadecanoids & 7 & 293.2122 & 2.10 & 0.44 & Yes \\
\hline 9-OxoODE & & & & 2.13 & 6.29 & Yes \\
\hline 13-HOTrE & & & & 2.72 & & Yes \\
\hline 15-HEPE & Hydroxy/hydroperoxyeicosapentaenoic & 8 & 317.2122 & 2.69 & 4.09 & Yes \\
\hline 5-HEPE & acids & & & 3.23 & & Yes \\
\hline 13-HODE & Other octadecanoids & 9 & 295.2279 & 2.68 & 1.48 & Yes \\
\hline 9-HODE & & & & 2.85 & & Yes \\
\hline 11,12-EET & Epoxyeicosatrienoic & 10 & 319.2279 & 1.70 & 1.09 & No \\
\hline 5,6-EET & acids & & & 1.84 & 6.18 & No \\
\hline 12-HETE & Hydroxy/hydroperoxyeicosatetraenoic & & & 2.54 & 0.96 & Yes \\
\hline 15-HETE & acids & & & 2.64 & 0.90 & Yes \\
\hline 11-HETE & & & & 2.75 & 2.76 & Yes \\
\hline 5-HETE & & & & 3.14 & & Yes \\
\hline 14-HDoHE & Docosanoids & 11 & 343.2279 & 2.90 & 3.78 & Yes \\
\hline 4-HDoHE & & & & 3.43 & & Yes \\
\hline 15-HETrE & Hydroxy/hydroperoxyeicosatrienoic acids & - & 321.2435 & 2.78 & & Yes \\
\hline$\gamma$-Linolenic acids & Unsaturated fatty acids & 12 & 277.2173 & 1.27 & 0.42 & Yes \\
\hline$\alpha$-Linolenic acids & & & & 1.30 & & Yes \\
\hline
\end{tabular}




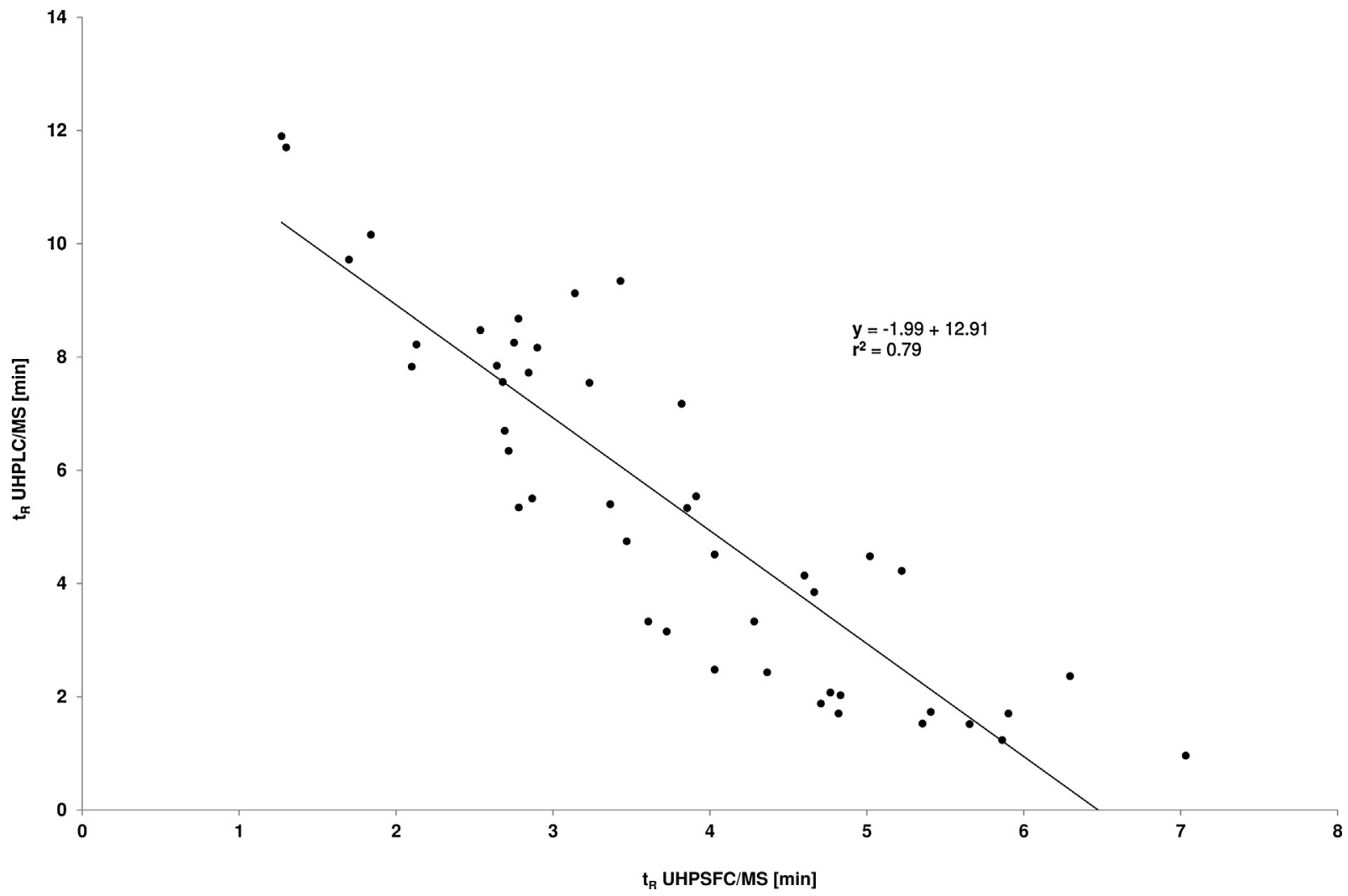

Fig. 8. Correlation of retention times in UHPLC/ESI-MS and UHPSFC/ESI-MS methods using final conditions.

position of double bond in the cyclopentane ring had higher contribution to the separation of isomers. For diastereoisomers PGF2 $\alpha$ and 8-iso-PGF2 $\alpha$ and functional isomers of 15-keto-PGF2 $\alpha$ and PGF2 $\alpha$, higher retention was observed in UHPSFC, and this was accompanied by higher resolution.

The sensitivity of given analytical method was also an important aspect due to low concentrations of oxylipins in biological samples. Approximately 3.5-fold higher sensitivity on average was observed in UHPLC/MS in comparison to UHPSFC/MS. Figs. 4 and 7 with the same scale of intensity illustrate the different sensitivity in both methods. In UHPSFC, the signal reduction could be attributed to the following reasons: 1 /applied higher flow rate, 2 /lower ionization efficiency due to decreased sample solubility during carbon dioxide expansion, and 3/dilution and splitting of eluted sample prior to MS detection [35]. The lower concentration of compounds in the effluent might be caused by different diameters between UHPSFC (3 mm) and UHPLC (2.1 mm) columns [36].

\subsection{Identification of oxylipins in human plasma}

The enrichment of oxylipins through polar and nonpolar matrix removal is a critical step prior to their analysis due to their trace concentration in biological samples. Nowadays, SPE is one of the most efficient and widespread technique in the sample preparation procedure. The polymeric StrataX RP column showed a good recovery for oxylipins $[7,17,24,37]$. The application of relatively high volume of plasma sample was needed due to the limited injection volume in UHPSFC. Numerous glycerophospholipids (PL), mainly lyso species, enriched with oxylipins and fatty acids in the extracts, caused matrix effects due to the coelution with oxylipins, which eluted in the range of 2-7 min in UHPSFC and 1-10 min in UHPLC (Tables 2 and 3). This unfavorable effect was more pronounced in UHPLC owing to comparable retention of oxylipins and LPLs in the RP separation. For example, high abundant LPI 20:4 and LPI 18:2, coeluted with PGE2 and PGD2 at $2.1 \mathrm{~min}$. Retention times of other LPE and LPC were found in the range of 8-10 min, e.g., LPE 18:2, LPE 22:6, LPC 16:0, and LPC 22:6, while in UHPSFC they eluted after $9 \mathrm{~min}$, which reduces the risk of ion suppression effects. Fatty acids had higher retention times in UHPLC (after $9.5 \mathrm{~min}$ ) and lower retention in UHPSFC (before $2.3 \mathrm{~min}$ ), therefore the risk of ion suppression effects is relatively low in both cases.

Tables 2 and 3 show 31 oxylipins identified in human plasma based on 48 standards by both methods. Considering better separation of isomers and higher sensitivity, the optimized UHPLC/MS method was selected for the identification of additional plasma oxylipins. The further identification of additional oxylipins without available authentic standards was based on their retention times, accurate masses of precursor ions ( $7 \mathrm{ppm}$ mass tolerance at maximum), and related fragment ions using homemade database created based on own measurements and literature sources [1-7,12,16-18,23,38,39]. Relevant chromatographic and MS data of additional 20 possible compounds in plasma including retention times, $m / z$ of precursor ions, and observed fragment ions corresponding to structures are given in Table 4.

\section{Conclusions}

Optimized UHPLC/ESI-MS and UHPSFC/ESI-MS methods have been applied for the analysis of 46 oxylipins and 2 fatty acids standards. In UHPLC, mobile phase additives and their concentrations significantly influence the chromatographic retention and MS sensitivity, especially the use of acetic acid brings advantages over ammonium acetate or formate in terms of the resolution and sensitivity. The UHPSFC/ESI-MS method for oxylipins is reported for the first time here. 1-AA column yields a better separation of oxylipins than other dedicated sub-2 $\mu \mathrm{m}$ UHPSFC stationary phases (2-PIC, 1-DIOL, DEA, and HSS C18). The chromatographic resolution of 
Table 4

UHPLC/MS/MS data for tentatively identified oxylipins without given standards in the human plasma sample.

\begin{tabular}{|c|c|c|c|c|c|c|}
\hline Name & Abbreviation & Subclass & $\begin{array}{l}\text { Experimental } \\
m / z \text { of } \\
{[\mathrm{M}-\mathrm{H}]^{-}}\end{array}$ & $\begin{array}{l}\text { Mass } \\
\text { accuracy } \\
{[\mathrm{ppm}]}\end{array}$ & $\begin{array}{l}\mathrm{t}_{\mathrm{R}} \text { in } \\
\text { UHPLC/MS } \\
\text { [min] }\end{array}$ & $m / z$ of observed fragment ions \\
\hline 5,9S,11R-trihydroxy-6E,14Z-prostadienoic acid-cyclo[8S,12R] & 5-iso-PGF2 $\alpha$ VI & Isoprostanes & 353.2317 & 4.8 & 1.89 & $335,317,309,115$ \\
\hline 9-oxo-11S,15S-dihydroxy-5Z,13E-prostadienoic acid & 11ß-PGE2 & Prostaglandins & 351.2164 & 3.7 & 1.95 & $333,315,271,189$ \\
\hline 9,10-dihydroxy-12Z-octadecenoic acid & 9,10-DiHOME & Other octadecanoids & 313.2371 & 4.2 & 5.14 & $295,277,201,177$ \\
\hline 9-hydroxy-10E,12Z,15Z-octadecatrienoic acid & 9-HOTrE & & 293.2106 & 5.5 & 6.20 & $275,231,185,171,121$ \\
\hline (+/-)-11-hydroxy-5Z,8Z,12E,14Z,17Z-eicosapentaenoic acid & 11-HEPE & Hydroxy/hydroperoxyeicosapentaenoic acids & 317.2115 & 2.2 & 6.28 & $\begin{array}{l}299,259,255,215,195,167 \\
149,121\end{array}$ \\
\hline (+/-)-14(15)-epoxy-5Z,8Z,11Z,17Z-eicosatetraenoic acid & 14(15)-EpETE & Other eicosanoids & 317.2106 & 5.0 & 6.58 & $299,255,219,207$ \\
\hline$(+\mid-)$-20-hydroxy-4Z,7Z,10Z,13Z,16Z,18E-docosahexaenoic acid & 20-HDoHE & Docosanoids & 343.2280 & -0.3 & 7.51 & $\begin{array}{l}325,299,285,281,241,187 \\
159,133,107\end{array}$ \\
\hline (+/-)-17-hydroxy-4Z,7Z,10Z,13Z,15E,19Z-docosahexaenoic acid & 17-HDoHE & & 343.2276 & 0.9 & 8.02 & $\begin{array}{l}325,281,273,245,229,227 \\
201,173,147,121,111\end{array}$ \\
\hline 15-oxo-5Z,8Z,11Z,13E-eicosatetraenoic acid & 15-oxo-ETE & Hydroxy/hydroperoxyeicosatetraenoic acids & 317.2107 & 4.7 & 8.05 & $299,273,139,113$ \\
\hline (+/-)-9,10-epoxy-12Z-octadecenoic acid & 9(10)-ЕрОМЕ & Other octadecanoids & 295.2271 & 2.7 & 8.20 & $277,183,201,171$ \\
\hline$(+/-)$-10-hydroxy-4Z,7Z,11E,13Z,16Z,19Z-docosahexaenoic acid & 10-HDoHE & Docosanoids & 343.2278 & 0.3 & 8.22 & $\begin{array}{l}325,299,281,227,181,161 \\
153,121\end{array}$ \\
\hline (+/-)-11-hydroxy-4Z,7Z,9E,13Z,16Z,19Z-docosahexaenoic acid & 11-HDoHE & & 343.2277 & 0.6 & 8.41 & $\begin{array}{l}325,281,227,194,165,149 \\
133,121,95\end{array}$ \\
\hline 8-hydroxy-5Z,9E,11Z,14Z-eicosatetraenoic acid & 8-HETE & Hydroxy/hydroperoxyeicosatetraenoic acids & 319.2276 & 0.9 & 8.60 & $301,257,163,155$ \\
\hline (+/-)-7-hydroxy-4Z,8E,10Z,13Z,16Z,19Z-docosahexaenoic acid & 7-HDoHE & Docosanoids & 343.2278 & 0.3 & 8.61 & $\begin{array}{l}325,281,245,227,201,147 \\
141,121,113,97\end{array}$ \\
\hline (+/-)-8-hydroxy-4Z,6E,10Z,13Z,16Z,19Z-docosahexaenoic acid & 8-HDoHE & & 343.2276 & 0.9 & 8.69 & $299,281,243,189,135,109$ \\
\hline 9-hydroxy-5Z,7E,11Z,14Z-eicosatetraenoic acid & 9-HETE & $\begin{array}{l}\text { Hydroxy/hydroperoxyeicosatetraenoic } \\
\text { acids }\end{array}$ & 319.2272 & 2.2 & 8.77 & $\begin{array}{l}301,275,257,229,203,179 \\
167,139,123,69\end{array}$ \\
\hline 12-oxo-5Z,8Z,10E,14Z-eicosatetraenoic acid & 12-oxo-ETE & & 317.2122 & 0.0 & 9.01 & $299,273,235,153$ \\
\hline (6E,8Z,11Z)-5-hydroxyicosa-6,8,11-trienoic acid & 5-HETrE & & 321.2415 & 6.2 & 9.10 & $303,259,205,115$ \\
\hline (+/-)-12(13)-epoxy-9Z-octadecenoic acid & 12(13)-ЕрОМЕ & Other octadecanoids & 295.2275 & 1.4 & 9.20 & $277,195,183$ \\
\hline 5-oxo-6E,8Z,11Z,14Z-eicosatetraenoic acid & 5-OXo-ETE & Hydroxy/hydroperoxyeicosatetraenoic acids & 317.2102 & 6.3 & 9.63 & $299,273,245,203,129$ \\
\hline
\end{tabular}


several critical isomeric pairs in UHPSFC is lower compared to RPUHPLC, which shows that RP-like mode in UHPSFC is not really competitive to RP-UHPLC unlike to HILIC mode, where UHPSFC is superior to HILIC-UHPLC. This is probably caused by natural limitations of UHPSFC, where the typical mobile phase is nonpolar $\mathrm{CO}_{2}$ with the addition of some polar modifier, which is far from the typical RP mobile phases used in UHPLC. In general, the sensitivity of UHPLC/MS is higher by a factor of ca. 3.5 times in comparison to UHPSFC/MS, but some opposite examples can be found as well, e.g., prostaglandins. The sensitivity decrease is partially caused by different column diameters (the ratio of squared internal diameters is about 2). Finally, 31 oxylipins from 46 available standards are detected in the human plasma extract together with additional 20 tentatively identified endogenous oxylipins based on UHPLC/MS/MS measurements and home-made database of precursor and product ions and accurate $m / z$ values of measured ions.

\section{Acknowledgements}

This work was supported by the ERC CZ grant project LL1302 sponsored by the Ministry of Education, Youth and Sports of the Czech Republic. Róbert Berkecz thanks for the financial support to the János Bolyai Research Scholarship of the Hungarian Academy of Sciences.

\section{Appendix A. Supplementary data}

Supplementary data associated with this arti-
cle can be found, in the online version, at
http://dx.doi.org/10.1016/j.chroma.2017.06.070.

\section{References}

[1] V.B. O’Donnel, B. Maskrey, G.W. Taylor, Eicosanoids: generation and detection in mammalian cells, in: B. Larijani, R. Woscholski, C.A. Rosser (Eds.), Lipid Signaling Protocols, Humana Press, New York, 2009, pp. 5-24.

[2] D. Wang, R.N. DuBois, Measurement of eicosanoids in cancer tissues, in: H.A. Brown (Ed.), Methods in Enzymology, vol. 433, Elsevier, 2007, pp. 27-50.

[3] D. Wang, R.N. DuBois, Eicosanoids and cancer, Nat. Rev. Cancer 10 (2010) $181-193$.

[4] E.A. Dennis, P.C. Norris, Eicosanoid storm in infection and inflammation, Nat Rev. Immunol. 15 (2015) 511-523.

[5] H. Tapiero, G. Nguyen Ba, P. Couvreur, K.D. Tew, Polyunsaturated fatty acids (PUFA) and eicosanoids in human health and pathologies, Biomed. Pharmacother. 56 (2002) 215-222.

[6] R. Loomba, O. Quehenberger, A. Armando, E.A. Dennis, Polyunsaturated fatty acid metabolites as novel lipidomic biomarkers for noninvasive diagnosis of nonalcoholic steatohepatitis, J. Lipid Res. 56 (2015) 185-192.

[7] O. Quehenberger, A.M. Armando, A.H. Brown, S.B. Milne, D.S. Myers, A.H. Merrill, S. Bandyopadhyay, K.N. Jones, S. Kelly, R.L. Shaner, C.M. Sullards, E. Wang, R.C. Murphy, R.M. Barkley, T.J. Leiker, C.R. Raetz, Z. Guan, G.M. Laird D.A. Six, D.W. Russell, J.G. McDonald, S. Subramaniam, E. Fahy, E.A. Dennis, Lipidomics reveals a remarkable diversity of lipids in human plasma, J. Lipid Res. 51 (2010) 3299-3305.

[8] M. Puppolo, D. Varma, S.A. Jansen, A review of analytical methods for eicosanoids in brain tissue, J. Chromatogr. B 964 (2014) 50-64.

[9] C. Ferreiro-Vera, J.M. Mata-Granados, F. Priego-Capote, J.M. Quesada-Gómez, M.D. Luque de Castro, Automated targeting analysis of eicosanoid inflammation biomarkers in human serum and in the exometabolome of stem cells by SPE-LC-MS/MS, Anal. Bioanal. Chem. 399 (2011) 1093-1103.

[10] R.C. Murphy, R.M. Barkley, K.Z. Berry, K.Z. Berry, J. Hankin, K. Harrsion, C. Johnson, J. Krank, A. McAnoy, C. Uhlson, S. Zarini, Electrospray ionization and tandem mass spectrometry of eicosanoids, Anal. Biochem. 346 (2005) 1-42.

[11] K.R. Maddipati, R. Romero, T. Chaiworapongsa, Sen-Lin Zhou, Z. Xu, A.L. Tarca, J.P. Kusanovic, H. Munoz, K.V. Honn, Eicosanomic profiling reveals dominance of the epoxygenase pathway in human amniotic fluid at term in spontaneous labor, The FASEB J. Res. Commun. 28 (2016) 4835-4846.

[12] D.D. Shinde, K.B. Kim, K.S. Oh, N. Abdalla, K.H. Liu, S.K. Bae, J.H. Shon, H.S. Kim, D.-H. Kim, J.G. Shin, LC-MS/MS for the simultaneous analysis of arachidonic acid and 32 related metabolites in human plasma: basal plasma concentrations and aspirin-induced changes of eicosanoids, J. Chromatogr. B 911 (2012) 113-124.

[13] T. Nishisho, T. Tonai, Y. Tamura, T. Ikata, Experimental and clinical studies of eicosanoids in cerebrospinal fluid after spinal cord injury, Neurosurgery 39 (1996) 950-957.
[14] K. Sterz, G. Scherer, J. Ecker, A simple and robust UPLC-SRM/MS method to quantify urinary eicosanoids, J. Lipid Res. 53 (2012) 1026-1036.

[15] J. Song, X. Liu, J. Wuc, M.J. Meehan, J.M. Blevitt, P.C. Dorrestein, M.E. Milla, A highly efficient, high-throughput lipidomics platform for the quantitative detection of eicosanoids in human whole blood, Anal. Biochem. 433 (2015) 181-188.

[16] K. Strassburg, A.M.L. Huijbrechts, K.A. Kortekaas, J.H. Lindeman, T.L. Pedersen, A. Dane, R. Berger, A. Brenkman, T. Hankemeier, J. van Duynhoven, E. Kalkhoven, J.W. Newman, R.J. Vreeken, Quantitative profiling of oxylipins through comprehensive LC-MS/MS analysis: application in cardiac surgery, Anal. Bioanal. Chem. 404 (2012) 1413-1426.

[17] Y. Wang, A.M. Armando, O. Quehenberger, C. Yan, E.A. Dennis, Comprehensive ultra-performance liquid chromatographic separationand mass spectrometric analysis of eicosanoid metabolites in human samples, J. Chromatogr. A 1359 (2014) 60-69.

[18] A.F. Galvão, T. Petta, N. Flamand, V.R. Bollela, C.L. Silva, L.R. Jarduli, K.C.R. Malmegrim, B.P. Simões, L.A.B. de Moraes, L.H. Faccioli, Plasma eicosanoid profiles determined by high-performance liquid chromatography coupled with tandem mass spectrometry in stimulated peripheral blood from healthy individuals and sickle cell anemia patients in treatment, Anal. Bioanal. Chem. 408 (2016) 3613-3623.

[19] L. Nováková, A.G.G. Perrenoud, I. Francois, C. West, E. Lesellier, D. Guillarme Modern analytical supercritical fluid chromatography using columns packed with sub-2 $\mu \mathrm{m}$ particles: a tutorial, Anal. Chim. Acta 824 (2014) 18-35.

[20] T. Berg, L. Kaur, A. Risnes, S.M. Havig, R. Karinen, Determination of a selection of synthetic cannabinoids and metabolites in urine by UHPSFC-MS/MS and by UHPLC-MS/MS, Drug Test. Anal. 8 (2016) 708-722.

[21] A.G.G. Perrenoud, J.L. Veuthey, D. Guillarme, Comparison of ultra-high performance supercritical fluid chromatography and ultra-high performance liquid chromatography for the analysis of pharmaceutical compounds, J. Chromatogr. A 1266 (2012) 158-167.

[22] M. Lísa, M. Holčapek, High-throughput and comprehensive lipidomic analysis using ultrahigh-performance supercritical fluid chromatography-mass spectrometry, Anal. Chem. 87 (2015) 7187-7195.

[23] M. Sud, E. Fahy, D. Cotter, A. Brown, E. Dennis, C. Glass, R. Murphy, C. Raetz, D. Russell, S. Subramaniam, LMSD: LIPID MAPS structure database, Nucleic Acids Res. 35 (2007) 527-532.

[24] A.I. Ostermann, Ina Willenberg, N.H. Schebb, Comparison of sample preparation methods for the quantitative analysis of eicosanoids and other oxylipins in plasma by means of LC-MS/MS, Anal. Bioanal. Chem. 407 (2015) 1403-1414

[25] M. Mal, P.K. Koh, P.Y. Cheah, E.C.Y. Chan, Ultra-pressure liquid chromatography/tandem mass spectrometry targeted profiling of arachidonic acid and eicosanoids in human colorectal cancer, Rapid Commun. Mass Spectrom. 25 (2011) 755-764.

[26] J. McMurry, Biomolecules: amino acids, peptides, and proteins, in: J. McMurry (Ed.), Organic Chemistry, ninth edition, Cengage Learning, Boston, 2016, pp. 870-907.

[27] M. Müller, T.C. Sorrell, Quantitation of sulfidopeptide leukotrienes by reversed-phase high-performance liquid chromatography, J. Chromatogr. 343 (1985) 213-218.

[28] H.A. Claessens, A comparative study of test methods for stationary phases for reversed-phase column in HPLC, in: H.A. Claessens (Ed.), Characterization of Stationary Phases for Reversed-phase Liquid Chromatography: Column Testing, Classification and Chemical Stability, Technische Universiteit Eindhoven, Eindhoven, 1999, pp. 107-142.

[29] V. Desfontaine, J.L. Veuthey, D. Guillarme, Evaluation of innovative stationary phase ligand chemistries and analytical conditions for the analysis of basic drugs by supercritical fluid chromatography, J. Chromatogr. A 1438 (2016) 244-253.

[30] K. Hettiarachchi, A. Yun, M. Kong, J.R. Jacobsen, Q. Xue, The use of SFC in discovery sciences, in: G.K. Webster (Ed.), Supercritical Fluid Chromatography: Advances and Applications in Pharmaceutical Analysis, CRC Press Taylor \& Francis Group, Boca Raton, 2014, pp. 15-41.

[31] J.W. Caldwell, W.B. Caldwell, G.K. Webster, Z. Wang, Method development of achiral SFC, in: G.K. Webster (Ed.), Supercritical Fluid Chromatography: Advances and Applications, in: Pharmaceutical Analysis, CRC Press Taylor \& Francis Group, Boca Raton, 2014, pp. 65-96.

[32] C. Mantell, M. Rodríguez, E. Martínez de la Ossa, Measurement of the diffusion coefficient of a model food dye (malvidin 3,5-diglucoside) in a high pressure $\mathrm{CO} 2+$ methanol system by the chromatographic peak-broadening technique, J. Supercrit. Fluids 25 (2003) 57-68.

[33] T.A. Berger, Physical chemistry of mobile phases used in packed column SFC, in: R.M. Smith (Ed.), Packed Column SFC, The Royal Society of Chemistry, Cambridge, 1995, pp. 43-71.

[34] J.G. Bollinger, W. Thompson, Y. Lai, R.C. Oslund, T.S. Hallstrand, M. Sadilek, F. Turecek, M.H. Gelb, Improved sensitivity mass spectrometric detection of eicosanoids by charge reversal derivatization, Anal. Chem. 82 (2010) 6790-6796.

[35] Y. Zhao, SFC in process analytical chemistry, in: G.K. Webster (Ed.), Supercritical Fluid Chromatography: Advances and Applications in Pharmaceutical Analysis, CRC Press Taylor \& Francis Group, Boca Raton, 2014, pp. 195-224.

[36] V. Desfontaine, L. Nováková, F. Ponzetto, R. Nicoli, M. Saugy, J.L. Veuthey, D. Guillarme, Liquid chromatography and supercritical fluid chromatography as 
alternative techniques to gas chromatography for the rapid screening of anabolic agents in urine, J. Chromatogr. A 1451 (2016) 145-155.

[37] R. Deems, M.W. Buczynski, R. Bowers-Gentry, R. Harkewicz, E.A. Dennis,

Detection and quantitation of eicosanoids via high performance liquid

chromatography-electrospray ionization-mass spectrometry, in: H.A. Brown

(Ed.), Methods in Enzymology, vol. 432, Elsevier, 2007, pp. 59-82.
[38] R.C. Murphy, Eicosanoid and bioactive lipid mediators, in: R.C. Murphy (Ed.), Tandem Mass Spectrometry of Lipids, Molecular Analysis of Complex Lipids, The Royal Society of Chemistry, Cambridge, 2015, pp. 40-74.

[39] R.C. Murphy, R.M. Barkley, K.Z. Berry, J. Hankin, K. Harrison, C. Johnson, J. Krank, A. McAnoy, C. Uhlson, S. Zarini, Electrospray ionization and tandem mass spectrometry of eicosanoids, Anal. Biochem. 346 (2005) 1-42. 